\title{
Numerical investigation of spontaneous flame propagation under RCCI conditions
}

\author{
Ankit Bhagatwala ${ }^{\mathrm{a}, *}$, Ramanan Sankaran ${ }^{\mathrm{b}}$, Sage Kokjohn ${ }^{\mathrm{c}}$, Jacqueline H. Chen ${ }^{\mathrm{a}}$ \\ ${ }^{a}$ Combustion Research Facility, Sandia National Laboratories, Livermore, CA 94550 \\ ${ }^{b}$ National Center for Computational Sciences, Oak Ridge National Laboratories, Oak Ridge, TN 37831, \\ USA \\ ${ }^{c}$ Department of Mechanical Engineering, University of Wisconsin-Madison, 1500 Engineering Dr, \\ Madison, WI 53706, USA
}

\begin{abstract}
This paper presents results from one and two-dimensional direct numerical simulations under Reactivity Controlled Compression Ignition (RCCI) conditions of a primary reference fuel (PRF) mixture consisting of n-heptane and iso-octane. RCCI uses in-cylinder blending of two fuels with different autoignition characteristics to control combustion phasing and the rate of heat release. These simulations employ an improved model of compression heating through mass source/sink terms developed in a previous work by Bhagatwala et al. [22], which incorporates feedback from the flow to follow a predetermined experimental pressure trace. Two-dimensional simulations explored parametric variations with respect to temperature stratification, pressure profiles and n-heptane concentration. Statistics derived from analysis of diffusion/reaction balances locally normal to the flame surface were used to elucidate combustion characteristics for the different cases. Both deflagration and spontaneous ignition fronts were observed to co-exist, however it was found that higher n-heptane
\end{abstract}

\footnotetext{
${ }^{*}$ Corresponding author

Email address: abhagat@sandia.gov (Ankit Bhagatwala)
}

Preprint submitted to Combustion and Flame

April 8, 2015 
concentration provided a greater degree of flame propagation, whereas lower n-heptane concentration (higher fraction of iso-octane) resulted in more spontaneous ignition fronts. A significant finding was that simulations initialized with a uniform initial temperature and a stratified n-heptane concentration field, resulted in a large fraction of combustion occurring through flame propagation. It was also found that the proportion of spontaneous ignition fronts increased at higher pressures due to shorter ignition delay when other factors were held constant. For the same pressure and fuel concentration, the contribution of flame propagation to the overall combustion was found to depend on the level of thermal stratification, with higher initial temperature gradients resulting in more deflagration and lower gradients generating more ignition fronts. Statistics of ignition delay are computed to assess the Zeldovich [34] theory for the mode of combustion propagation based on ignition delay gradients.

Keywords RCCI, thermal stratification, reactivity stratification, premixed flame, autoignition

Notice: This manuscript has been authored by UT-Battelle, LLC, under Contract No. DEAC0500OR22725 with the U.S. Department of Energy. The United States Government retains and the publisher, by accepting the article for publication, acknowledges that the United States Government retains a non-exclusive, paid-up, irrevocable, world-wide license to publish or reproduce the published form of this manuscript, or allow others to do so, for the United States Government purposes. 


\section{Introduction}

Recently, several advanced combustion concepts have emerged which hold the promise of simultaneously improving engine efficiency, reducing emissions of $\mathrm{NO}_{\mathrm{X}}$ and soot, and allowing utilization of renewable fuels. These combustion strategies can generally be lumped into the category of dilute, premixed compression ignition (PCI) combustion. Examples of several promising PCI combustion strategies can be found in Manente et al. [1], Dec et al. [2], and Kokjohn et al. [3].

Highly dilute operation lowers combustion temperatures, yielding a $\mathrm{NO}_{\mathrm{X}}$ reduction due to the high activation energy of NO formation reactions. The high level of pre-combustion mixing associated with these combustion modes reduces rich regions and avoids soot formation. Furthermore, the lower combustion temperatures have been shown to reduce heat transfer losses and improve efficiency of heavy-duty engines by up to $10 \%$ over state-of-the-art diesel engines [3]. Finally, PCI has been shown to be well-suited for operation using alternative fuels (e.g., natural gas [4]) and renewable fuels (e.g., ethanol [5]). In fact, the benefits of PCI combustion are often enhanced due to the autoignition characteristics of common renewable fuels [6].

Single fuel premixed compression-ignition strategies are operationally limited to low loads due to difficulties controlling combustion phasing and heat release rate which, in turn, affects the pressure rise rate. Dual fuel strategies such as Reactivity-Controlled Compression Ignition (RCCI) use in-cylinder blending of two fuels with different autoignition characteristics to control combustion phasing and heat release rate. In the RCCI combustion mode, a homogeneous charge of a low-reactivity fuel (e.g., gasoline) is created using port-fuel-injection or early direct-injection. Later in the compression stroke, a high-reactivity fuel (e.g., diesel 
fuel) is injected into the combustion chamber using direct-injection.

Kokjohn et al. [7] used high-speed chemiluminescence imaging in an optically accessible engine to visualize the RCCI combustion process with iso-octane as a surrogate for the low-reactivity fuel and n-heptane as a surrogate for the high-reactivity fuel. Selected hightemperature combustion chemiluminescence images are shown in Figure 1. Small ignition pockets were initially observed in two locations, inside the edge of the piston bowl rim, and in the squish region (i.e., above the piston bowl-rim). These ignition zones were found to grow for several crank angles while more ignition pockets continued to appear. The separate ignition pockets then merged into larger, more coherent reaction zones as the reaction zone moved from the outer region of the chamber toward the center of the combustion chamber.

In the same study, Kokjohn et al. [7] used fuel tracer fluorescence imaging to relate the fuel distribution prior to ignition to the ignition locations and the preferred direction of reaction zone growth. Figure 2 shows ensemble averaged equivalence ratio and local octane number distributions derived from fuel tracer fluorescence imaging. These experiments showed that ignition occurs in the region containing the highest concentration of high-reactivity fuel (i.e., n-heptane). The reaction zone then grows following the gradient in fuel reactivity. Although the previously discussed optical engine experiments provide a qualitative description of the RCCI combustion event, the mechanism controlling the local growth of the auto-ignited zones is unclear.

There is currently only a limited fundamental understanding of the nature of combustion in a reactive mixture that is conducive to both spontaneous ignition front propagation and premixed flame propagation. Zeldovich [34] studied the combustion of a non-uniform mixture in several distinct regimes of propagation that include spontaneous ignition fronts and flame 


\section{Piston Crown Cylinder Head Window Images Window Images}
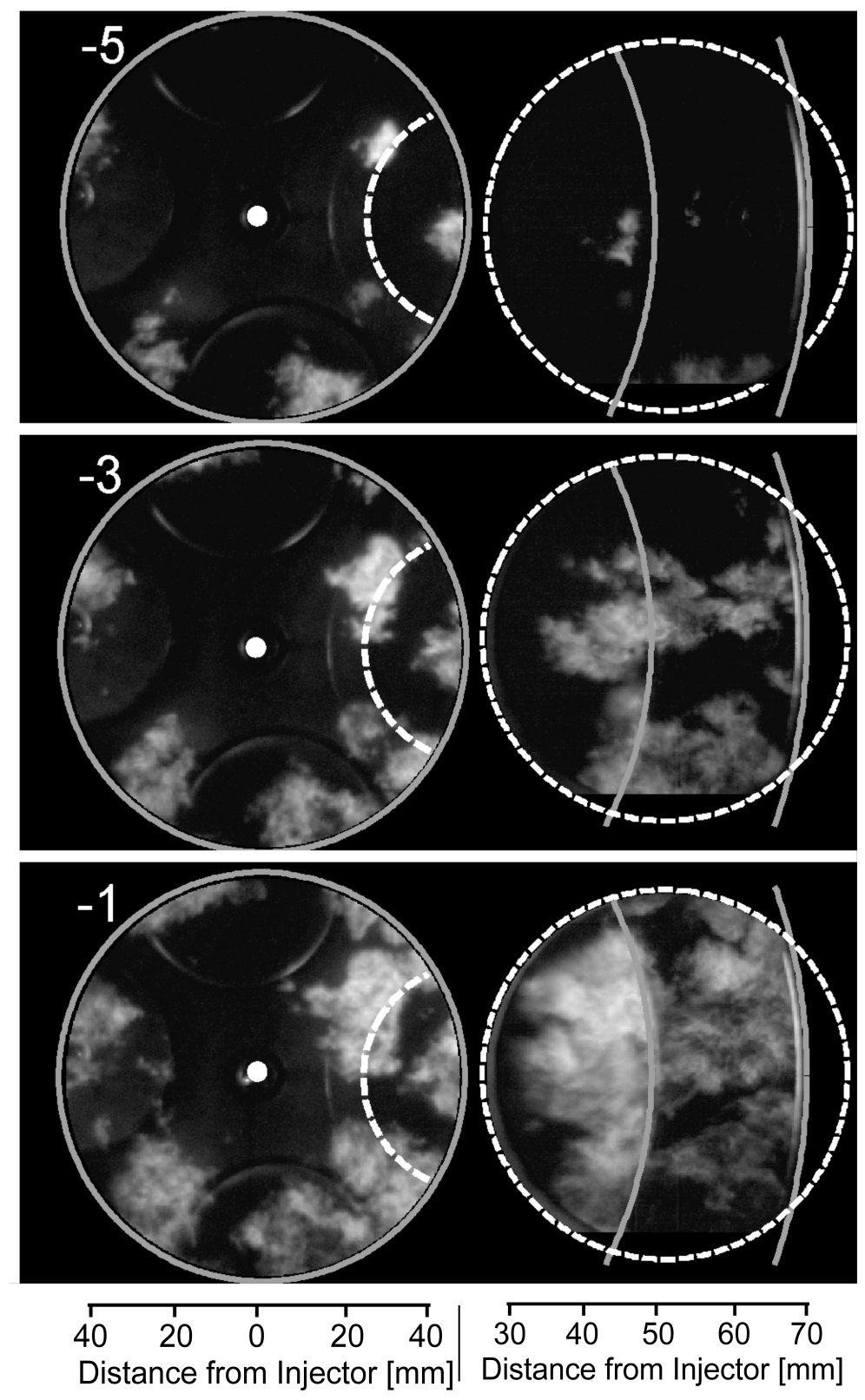

Figure 1: High-speed camera image sequence of RCCI combustion from Kokjohn et al. [7]. The images on the left-hand side were acquired with the high-speed camera viewing upward through the flat piston crown window, while the images on the right-hand side were acquired with the camera viewing downward through the cylinder head window. On the left-hand side images (piston crown), the location of the common-rail fuel injector is indicated by a white circle, the location of the piston bowl rim is indicated by the solid gray line, and the overlapping field-of-view of the cylinder gead window and piston crown window is indicated by the white dashed line. On the right-hand side images (cylinder head), the field-of-view of the cylinder head window is shown by the white dashed line, the left-most arc shows the location of the piston bowl rim, and the right-most arc shows the location of the cylinder liner. Note that two sets of images were not acquired simultaneously. 
propagation. He showed that the propagation speed of the ignition front and its transition between these two combustion modes is governed by the reactivity gradient. In a numerical study of autoignition in a thermally stratified mixture using direct numerical simulation (DNS) $[16,17,18]$, it was confirmed that the mode and speed of propagation is controlled by the local temperature gradient at the onset of combustion, which is modulated from its initial value due to turbulent mixing. In $[16,17,18]$, a homogenous mixture composition was assumed such that the reactivity gradient was determined by the temperature gradient alone. However, in the presence of mixture stratification, the reactivity gradient would depend on both temperature and composition gradients, which are subject to turbulent mixing and diffusion. A later DNS study with ethanol mixture [22] used the chemical explosive mode analysis (CEMA) to identify the different modes of combustion. The CEMA analysis used the instantaneous thermal and composition state to determine the mode of combustion and was shown to be effective in identifying the transition between the modes. In this study however, a more unambiguous, first-principles based approach involving the reaction-diffusion balance of a reacting scalar is used to identify the combustion mode.

Thus, the goal of this study is to extend the prior simulation studies and to quantify the relative roles of spontaneous ignition fronts and premixed flame propagation under RCCI conditions, where both thermal and composition stratification are present. Premixed flame propagation has been shown to occur in RCCI engine simulations and is conjectured to occur in real engines. However, it is difficult to observe or measure these flames in engine experiments due to a lack of spatial resolution; thus, high-fidelity simulations are currently the only means to study this phenomenon. Here, we perform Direct Numerical Simulations (DNS) of autoignition under RCCI conditions to quantify the characteristics and likelihood of flame propagation. At this juncture, it is important to clarify that, since there is no external heat source such as a spark in this study, the energy release in all the simulations shown here is 


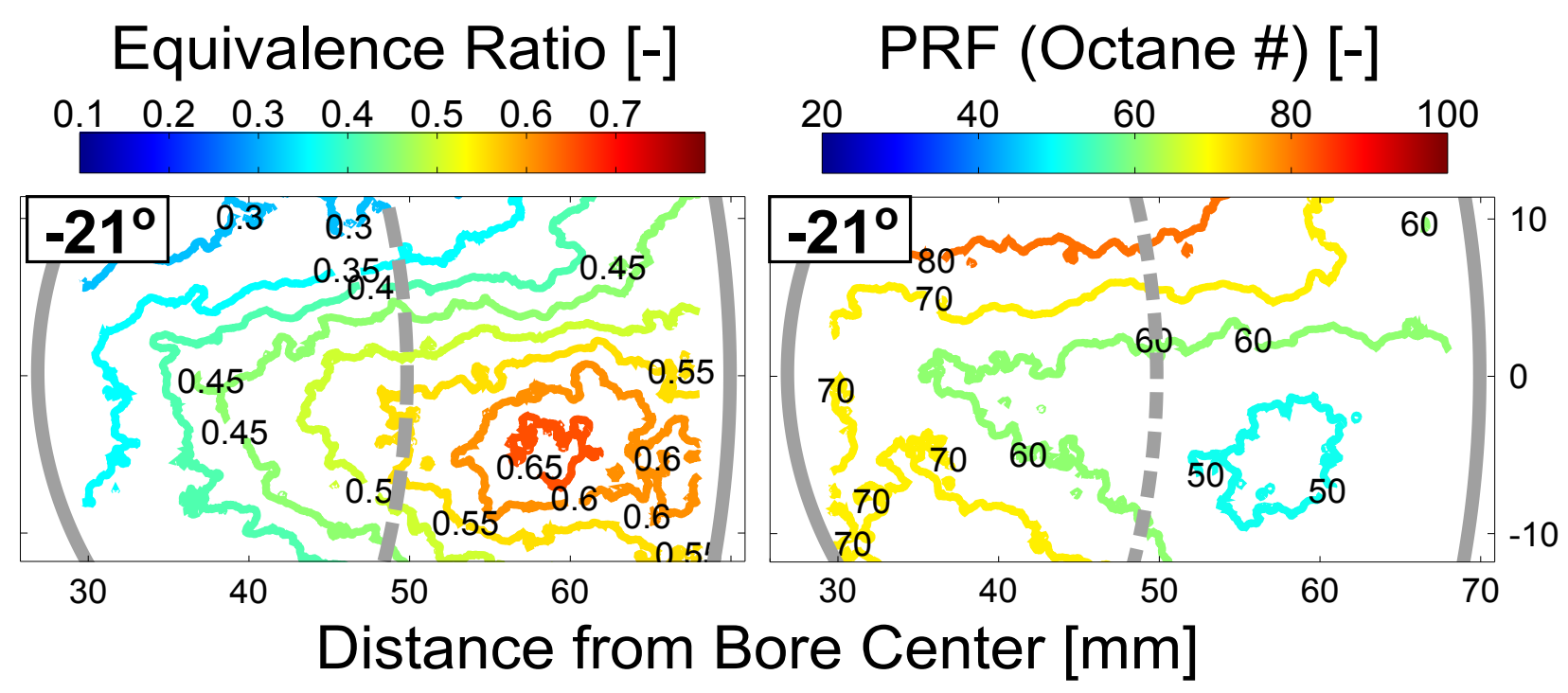

Figure 2: Equivalence ratio and PRF distributions for an RCCI case using premixed iso-octane and directinjected n-heptane from the experiments of Kokjohn et al. [7]. The field-of-view of the camera is the same as the images on the right-hand side of Figure 1 (i.e., viewing downward through the cylinder head window). The left-most solid gray line shows the inner edge of the camera field-of-view. The dashed gray line near the center of the images shows the location of the inner edge of the piston bowl rim. The right-most solid gray line shows the edge of the cylinder liner. 
triggered by autoignition by definition. The motivation behind this work is to study how the fronts emanating from the autoigniting kernels propagate, once they are formed. In other words, what role does transport, and in particular diffusion, play in the propagation of these kernels, if any. In that spirit, the term "spontaneous ignition fronts", henceforth referred to as "ignition fronts" for the sake of brevity, will be taken to mean a reaction-dominated ignition front, whereas a front in which reaction and diffusion are of comparable magnitude, will be termed a "flame" or "deflagration". A front speed is determined by the ignition delay differential between adjacent mixture parcels, which is directly related to the local scalar gradients in temperature and composition. Contrasting the role of flame propagation versus ignition front propagation is important for improving the predictive ability of models used in engine simulations.

Direct numerical simulation of autoignition under turbulent conditions has proved to be a valuable scientific tool for understanding the effects of turbulent mixing and aerodynamic straining on autoigniting kernels, especially in the presence of thermal and composition stratification $[18,22,24,12,25]$. However, the canonical configurations typically used in the DNS have several limitations that make the interpretation of the results difficult. DNS studies have been performed under constant volume conditions, which can adequately capture the ignition behavior in the cylinder at top dead center (TDC) conditions when there is no significant radical buildup or thermal runaway during the compression stroke [16, 17, 18]. However, it is also known that under the conditions of interest, there is significant radical buildup during the compression stroke due to the low temperature chemistry effects of hydrocarbon fuels. Therefore, the importance of capturing the radical buildup late in the compression stroke (i.e., near TDC) cannot be ignored. A previous DNS study by Domingo and Vervisch [29] and a recent DNS study of spark-assisted compression ignition with combustion phasing retard [22] used a mass source term to mimic the volume change and pressure 
rise within the combustion chamber due to piston motion.

A further limitation of previous DNS studies has been the small domain size used in the simulations compared to the size of the actual combustion chamber in an engine. While the simulations have been able to account for the microscale temperature and composition stratifications and gradients, they do not account for the large-scale thermal and composition stratifications that are present in a real engine chamber. These large-scale stratifications play a key role in smoothing and prolonging the pressure rise rate through two mechanisms. First, due to the large-scale stratifications, different regions of the chamber ignite at different times leading to a spreading of the heat release and consequent pressure rise. Second, the local pressure rise due to heat release propagates throughout the larger volume including the non-combusting spots. This causes compression heating of those regions while moderating the rate of pressure rise in the actively combusting region. This moderating effect is stronger when only a smaller fraction of the volume is generating heat at a time. Due to the small domain sizes used in DNS and the lack of large-scale stratifications, the fraction of volume generating heat is higher than under realistic conditions. Therefore, the rate of pressure rise and peak pressure observed in DNS are very different from experimental pressure traces at the same conditions. This poses serious difficulties when using the DNS to understand behavior observed in experiments. To alleviate this difficulty, we incorporate a mass injection model that allows the thermodynamic pressure of the DNS domain to be externally imposed to match the experimental pressure trace. This model extends the previous mass injection strategies [29, 22] by using the mass source term to not only account for volume changes due to piston motion, but to also replicate the pressure history of a fired engine. The mass injection model is described in section 2 .

In sections 4 and 5, we report results from one and two-dimensional DNS with a gaso- 
line primary reference fuel (PRF) blend of iso-octane and n-heptane. The two-dimensional DNS used two mass injection models, one that mimics the effects of volume change due to piston motion alone and a second model that replicates the pressure history observed experimentally. Parametric variations in fuel concentration, rate of compression and temperature stratification were studied to evaluate the roles of ignition fronts and premixed flame propagation under RCCI conditions.

\section{Formulation of pressure algorithm}

The pressure history observed in the experiment is modeled using a continuous and differentiable analytical function. Here, the analytical model is derived and the model constants are obtained by fitting against the experimental pressure trace as described below. We start with the slider-crank relation for the chamber volume [30]

$$
\frac{V}{V_{0}}=1+\frac{r-1}{2}\left[L+1-\cos \theta-\left(\mathrm{L}^{2}-\sin ^{2} \theta\right)^{1 / 2}\right]
$$

where $V_{0}$ is the clearance volume, $r$ is the compression ratio, $L$ is the ratio of connecting rod length to crank arm radius, and $\theta$ is the crank angle, which goes to zero at TDC. Close to TDC, Eq. 1 can be approximated as [31]

$$
\frac{V}{V_{0}}=1+g^{2} \pi^{2} \frac{\left(t-t_{0}\right)^{2}}{t_{\mathrm{c}}^{2}}
$$

where $g$ is a model factor to account for the crank slider geometry, $t_{0}$ is the time at TDC and $t_{\mathrm{c}}$ is the time taken for one crank rotation. For a polytropic compression coefficient of $n$, the motored pressure history close to TDC is given by,

$$
P_{\text {motored }}(t)=P_{0, \mathrm{~m}}\left[1+g^{2} \pi^{2} \frac{\left(t-t_{0}\right)^{2}}{t_{\mathrm{c}}^{2}}\right]^{-n}
$$


where, $P_{0, \mathrm{~m}}$ is the motored TDC pressure. Upon fitting the Equation 3 against the experimental motored pressure trace, the model constants are determined to be $g^{2}=13$ and $P_{0, \mathrm{~m}}=26.5$, given $t_{\mathrm{c}}=50 \mathrm{~ms}$. Also, $n$ is assumed to be 1.33. Equation 3 is then extended to account for pressure rise due to combustion near TDC as,

$$
P_{\text {combustion }}(t)=\Phi(t)\left[1+g^{2} \pi^{2} \frac{\left(t-t_{0}\right)^{2}}{t_{\mathrm{c}}^{2}}\right]^{-n}
$$

where

$$
\Phi(t)=\frac{1}{2}\left[P_{0, \mathrm{~m}}(1-E)+\left(P_{0, \mathrm{~m}}+P_{0, \mathrm{c}}\right)(1+E)\right]
$$

and

$$
E=\operatorname{erf}\left[\frac{\left(\mathrm{t}-\mathrm{t}_{0}-\mathrm{t}_{\mathrm{p}}\right)}{\sigma \sqrt{2}}\right],
$$

where $P_{0, \mathrm{c}}$ is the additional pressure due to combustion at TDC over and above the motored TDC pressure. $E$ can be thought of as a blending function that helps approximate the pressure profile under combustion by merging the pressure profile for the actual motored case and a hypothetical one with a peak pressure of $P_{0, \mathrm{~m}}+P_{0, \mathrm{c}}$. Upon fitting Equation 4 against the combusting pressure trace as shown in Figure 3 the model constants to account for pressure rise due to combustion, $P_{0, \mathrm{c}}$, were taken to be $28 \mathrm{bar}, t_{\mathrm{p}}$ was $0.5 \mathrm{~ms}$ before TDC, and $\sigma=0.7 \mathrm{~ms}$. It is seen from Figure 3 that a good agreement with the experimental trace can be obtained with these minimal model constants.

Given the analytical form of the pressure history, the DNS governing equations need to be modified with an appropriate mass source term to reproduce the prescribed pressure history. The fully compressible Navier-Stokes equations: continuity, momentum, total energy, and species continuity equations are solved in conservative form with detailed chemistry, 


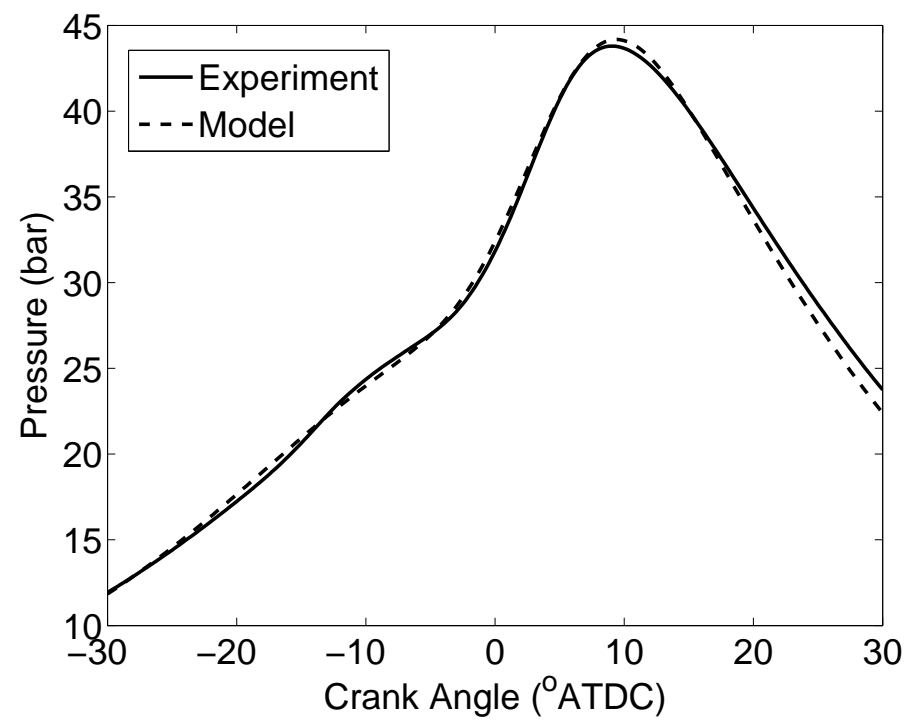

Figure 3: Experimental and modeled pressure history (non-motored) as a function of crank angle degrees. Experimental pressure trace taken from experiment by Kokjohn et. al [14] with n-heptane and iso-octane operating at 1200 RPM under low load conditions. A split n-heptane injection was used. The start-ofinjection (SOI) of the first pulse was at $-57 \mathrm{deg}$. ATDC and the SOI of the second pulse was at $-37 \mathrm{deg}$. CA50 point was $5^{\circ}$ ATDC. 


$$
\begin{aligned}
\frac{\partial \rho}{\partial t} & =-\frac{\partial \rho u_{i}}{\partial x_{i}}+\dot{m} \\
\frac{\partial \rho u_{i}}{\partial t} & =-\frac{\partial \rho u_{i} u_{j}}{\partial x_{j}}-\frac{\partial P}{\partial x_{i}}+\frac{\partial \tau_{i j}}{\partial x_{j}}+\dot{m} u_{\mathrm{i}} \\
\frac{\partial \rho e_{t}}{\partial t} & =-\frac{\partial \rho e_{t} u_{j}}{\partial x_{j}}-\frac{\partial P u_{j}}{\partial x_{j}}+\frac{\partial\left(\tau_{i j} \cdot u_{i}\right)}{\partial x_{j}}-\frac{\partial q_{j}}{\partial x_{j}}+\dot{m} e_{\mathrm{t}} \\
\frac{\partial \rho Y_{k}}{\partial t} & =-\frac{\partial \rho Y_{k} u_{j}}{\partial x_{j}}-\frac{\partial J_{k, j}}{\partial x_{j}}+\omega_{k}+\dot{m} Y_{k}
\end{aligned}
$$

where $\rho$ is the density, $u_{i}$ are the Cartesian velocity components, $P$ is pressure, $Y_{k}$ is the species mass fraction of the $k$ th species and $\omega_{k}$ is its reaction rate, $\tau_{i j}$ is the viscous stress tensor given by $\tau_{i j}=\mu\left(\frac{\partial u_{i}}{\partial x_{j}}+\frac{\partial u_{j}}{\partial x_{i}}-\frac{2}{3} \delta_{i j} \frac{\partial u_{l}}{\partial x_{l}}\right)$. The total specific energy is given by $e_{t}=u_{i} u_{i} / 2+h-P / \rho$ and the heat flux vector is given by $q_{i}=-\lambda \frac{\partial T}{\partial x_{i}}+\Sigma_{k} h_{k} J_{k, i}$. The species diffusive flux is computed through a mixture averaged formulation based on a form of Fick's law, $J_{k, i}=-\rho D_{k} \frac{\partial Y_{k}}{\partial x_{i}}-\frac{\rho D_{k} Y_{k}}{M} \frac{\partial M}{\partial x_{i}}$ where $M$ is the mixture-averaged molecular weight and $D_{k}$ is a mixture-averaged diffusion coefficient computed using the CHEMKIN transport package [9]. Nitrogen is used as the bath gas, i.e. the diffusion flux of nitrogen balances the sum of the diffusion fluxes for the other species in order to ensure that the net diffusion flux is zero.

The ideal gas equation of state is used such that

$$
P(\mathbf{x}, t)=P_{\mathrm{t}}(t)+p(\mathbf{x}, t)=\rho R T
$$

where $P_{\mathrm{t}}=\bar{P}$ is the mean thermodynamic pressure of the domain, $p$ is the local fluid dynamic pressure and $R$ is the specific gas constant. The bar (.) over a quantity indicates a spatial average computed over all points in the simulation domain. Upon, differentiating Eq. 8 and 
using equations 7 , the rate of change of pressure is determined to be,

$$
\frac{\partial P}{\partial t}=(\gamma-1)\left(\frac{\partial \rho e_{\mathrm{t}}}{\partial t}-u_{\mathrm{i}} \frac{\partial \rho u_{i}}{\partial t}+\frac{u_{\mathrm{i}}^{2}}{2} \frac{\partial \rho}{\partial t}-\sum_{k} h_{k} \frac{\partial \rho Y_{k}}{\partial t}\right)+\gamma R_{\mathrm{u}} T \sum_{k} \frac{1}{W_{k}} \frac{\partial \rho Y_{k}}{\partial t}
$$

where $R_{\mathrm{u}}$ is the universal gas constant and $W_{k}$ is the molecular weight of species $k$. The rate of change of pressure in the system expressed in eq. 9 can be decomposed into two components as,

$$
\frac{\partial P}{\partial t}=\left(\frac{\partial P}{\partial t}\right)_{\dot{m}=0}+\frac{\partial P_{\mathrm{m}}}{\partial t}
$$

where, the first term represents the innate rate of change of pressure in the system independent of mass injection. The second term represents the rate of change of pressure induced due to mass injection and is found by substituting the mass source term in Eq. 9 to be

$$
\frac{\partial P_{\mathrm{m}}}{\partial t}=(\gamma-1)\left(e_{\mathrm{t}} \dot{m}-\frac{u_{\mathrm{i}}^{2}}{2} \dot{m}-\sum_{\mathrm{k}} h_{\mathrm{k}} Y_{\mathrm{k}} \dot{m}\right) \cdot+\gamma R T \dot{m}
$$

Upon simplification,

$$
\frac{\partial P_{\mathrm{m}}}{\partial t}=\frac{P}{\rho} \dot{m}
$$

In order to have the system's mean thermodynamic pressure follow the prescribed pressure history, $\partial P_{\mathrm{m}} / \partial t$ has to equal the difference between the prescribed and the innate pressure change terms. Furthermore, the pressure change induced due to mass injection has to be uniform across the domain to avoid creating pressure gradients and undesirable influences on the fluid dynamics. Therefore,

$$
\frac{d P_{\mathrm{m}}}{d t}=\left(\frac{d P}{d t}\right)_{\text {prescribed }}-\overline{\left(\frac{\partial P}{\partial t}\right)_{\dot{m}=0}}
$$

where the prescribed rate of change of pressure is obtained by differentiating the analytical 
expression in eq. 4. Eq. 13 gives the required rate of pressure change through mass injection, which is uniform across the domain. The local mass source term is then obtained by rearranging Eq. 12 to give,

$$
\dot{m}=\frac{\rho}{P} \frac{d P_{\mathrm{m}}}{d t}
$$

which is then added to the governing equations (i.e. Eq. 7). This procedure allows the DNS to be conducted while precisely following the pressure history observed in the experiment.

\section{Numerical method}

The DNS code used in this study is called S3D, a massively parallel compressible reacting flow solver developed at Sandia National Laboratories [8]. The spatial derivatives are computed on an equi-spaced, finite difference grid using eighth-order centered finite differences [10]. A tenth order de-aliasing filter is applied every 10 time steps to remove high-wavenumber noise. The solution is integrated in time with a six-stage, fourth-order Runge-Kutta method [11]. For both one and two-dimensional simulations carried out here, periodic boundary conditions were used on all boundaries.

\subsection{Reduced gasoline primary reference fuel (PRF) mechanism}

The chemical kinetics of iso-octane $(\mathrm{PRF}=100)$ and $n$-heptane $(\mathrm{PRF}=0)$ are described by the reduced gasoline PRF mechanism of Luong et al. [12] (116 transported species and 861 reactions). This reduced mechanism was derived from a Lawrence Livermore National Laboratory (LLNL) detailed gasoline PRF mechanism [13]. The detailed mechanism, consisting of 874 species and 3796 reactions was reduced using a combination of directed relation graph (DRG)-based methods, isomer lumping, and timescale analysis. The reduced mechanism was validated for $\mathrm{PRF}$ mixtures ranging from neat n-heptane $(\mathrm{PRF}=0)$ to neat iso-octane 


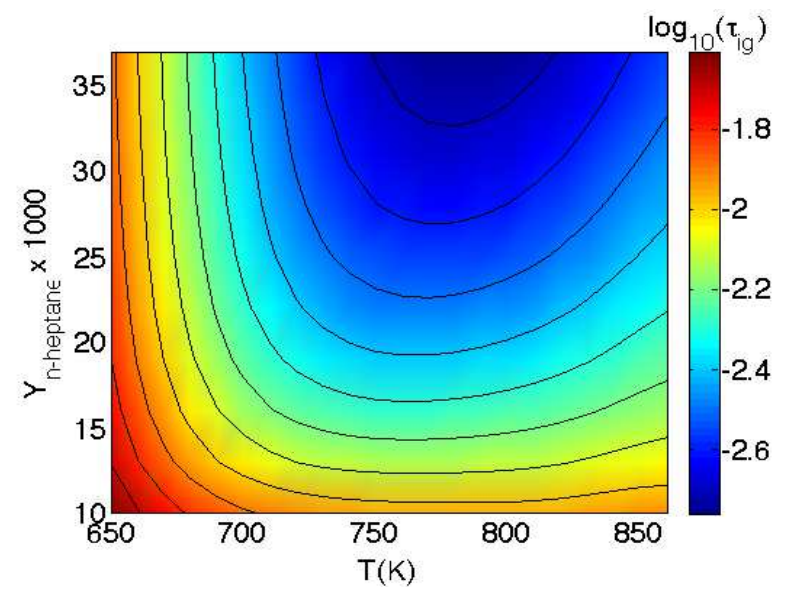

Figure 4: Zero-dimensional ignition delay map as a function of temperature and n-heptane concentration at a pressure of 30 bar. The quantity plotted is $\log _{10}\left(\tau_{i g}\right)$, where $\tau_{i g}$ is the homogeneous ignition delay time at constant volume obtained from 0-D Senkin simulations. Iso-octane concentration was kept constant at the same level as in the 2-D simulations, $Y_{\text {iso-octane }}=0.018$.

( $\mathrm{PRF}=100)$, by comparing ignition delay and extinction residence time. This validation was performed at pressures ranging from 1 atm to $50 \mathrm{~atm}$, equivalence ratios from 0.3 to 0.7 , and initial temperatures from $750 \mathrm{~K}$ to $1800 \mathrm{~K}[12]$.

Figure 4 shows the 0-D ignition delay at constant volume, spanning the parameter space at $t=0$ in the $1-\mathrm{D}$ and 2-D simulations presented in this study. Iso-octane concentration for these 0-D simulations was kept constant at the same level as in the 1-D and 2-D simulations presented later, so that $Y_{\text {iso-octane }}=0.018$. The negative temperature coefficient (NTC) behavior, denoted by the ignition delay isolines turning upwards beyond approximately $T=750 \mathrm{~K}$, is evident from this plot. Also noteworthy is the strong dependence of ignition delay on the concentration of n-heptane, especially at higher temperatures. This dependence is higher at lower concentrations of n-heptane, while showing asymptotic behavior at higher concentrations. 


\begin{tabular}{|c|c|c|c|c|c|c|}
\hline \hline Case \# & $\begin{array}{c}\Delta T \\
(K)\end{array}$ & $\begin{array}{c}\delta_{T} \\
(\mathrm{~mm})\end{array}$ & $\begin{array}{c}(d T / d x)_{\max } \\
(K / m m)\end{array}$ & $\Delta \phi_{n-\text { heptane }}$ & $\begin{array}{c}\delta_{n-\text { heptane }} \\
(\mathrm{mm})\end{array}$ & $\begin{array}{c}\text { Combustion } \\
\text { Mode }\end{array}$ \\
\hline \hline 1D-a & 50 & 0.8 & 140 & 0 & - & Flame \\
\hline 1D-b & 5 & 0.8 & 13 & 0 & - & Ignition front \\
\hline 1D-c & 0 & - & 0 & 0.23 & 0.8 & Flame \\
\hline
\end{tabular}

Table 1: Parameters for a Gaussian fluctuation in temperature and n-heptane equivalence ratio with respect to background values. Background values are $P=30$ bar, $T=714 K, \phi_{n-\text { heptane }}=0.32, \phi_{\text {iso-octane }}=0.27$. The background temperature value was chosen to be consistent with the engine experiments at -20 deg. ATDC (slightly before low temperature heat release was observed).

\subsection{Equivalence ratio computation}

Dual fuel configurations pose unique challenges in terms of defining mixture parameters such as mixture fraction or equivalence ratio. Here, we take the approach used in Kokjohn et al. [14], and define three types of equivalence ratios that will be used throughout the remainder of this paper. The equivalence ratio with respect to n-heptane and iso-octane are computed as, $\phi_{n-h e p t a n e}=\nu_{n h} Y_{n h, u} / Y_{O 2, u}$ and $\phi_{i s o-o c t a n e}=\nu_{i o} Y_{i o, u} / Y_{O 2, u}$, where subscripts 'nh' and 'io' represent n-heptane and iso-octane, respectively and $\nu$ is the stoichiometric oxygen-to-fuel mass ratio for a particular fuel [15]. An overall equivalence ratio is computed at every grid point based on the combined local fuel (n-heptane + iso-octane) and oxygen concentrations. This is equivalent to the equivalence ratio definition that is typically used in single fuel systems. The first two definitions assume that all of the local oxygen is available to react with both fuels, which is obviously not the case. Hence, they are not equivalence ratios in a strict sense, but should instead be considered as normalized concentrations of n-heptane or iso-octane.

\section{One dimensional simulations}

A series of highly resolved, one-dimensional simulations were performed to aid in the interpretation of the two-dimensional simulations presented in the next section. Table 1 shows 


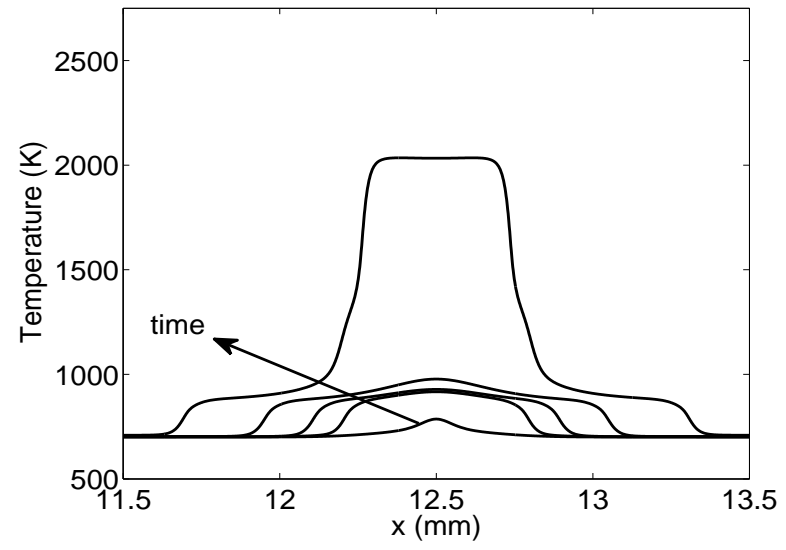

(a)

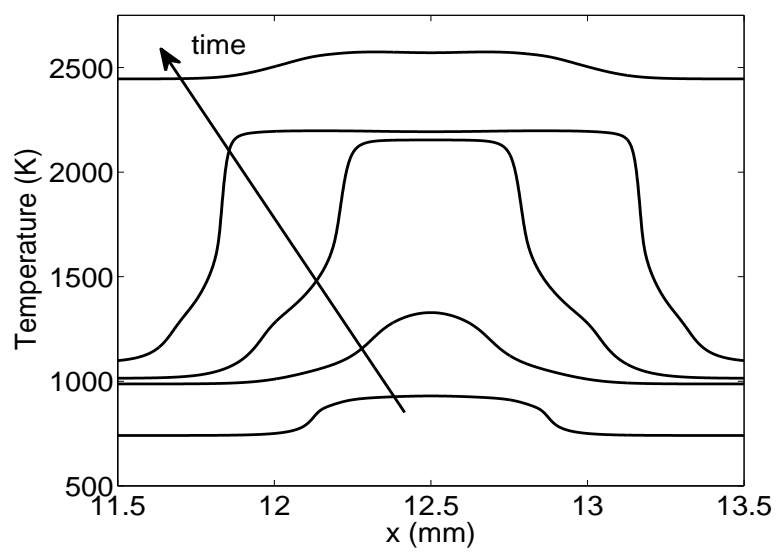

(b)

Figure 5: Evolution of the temperature profile for cases (a) 1D-a (b) 1D-b. The domain boundaries are $(0,25) \mathrm{mm}$. The domain has been truncated to show only the region of interest.

the physical parameters for the three cases simulated. A grid spacing of $\Delta x=1.25 \mu \mathrm{m}$ was used for all three cases. This grid spacing was chosen such that the thinnest radical layer had at least 15 grid points across it. Cases 1D-a and 1D-b were initialized with uniform composition and Gaussian temperature profiles with equal full widths at half maximum (FWHM) of $0.24 \mathrm{~mm}$ or $1 \%$ of the domain length, but different amplitudes. These initial conditions were chosen to test the sensitivity of the combustion mode to the local temperature gradient. Figure 5 shows the evolution of the temperature profiles for two cases with the equal fuel distributions, but different levels of temperature stratification. Case 1D-a (Figure 5(a)) has an initial temperature gradient of $140 \mathrm{~K} / \mathrm{mm}$ and case 1D-b has an initial temperature gradient of $13 \mathrm{~K} / \mathrm{mm}$. After ignition, the evolution of the temperature profile for the case with the higher initial temperature gradient (case 1D-a) has a similar appearance to a one-dimensional laminar flame. The case with the lower temperature initial temperature gradient appears to initially propagate as a premixed flame, but rapidly transitions to a reaction-driven ignition front. These observations were confirmed by plotting the reaction and diffusion rates of the 


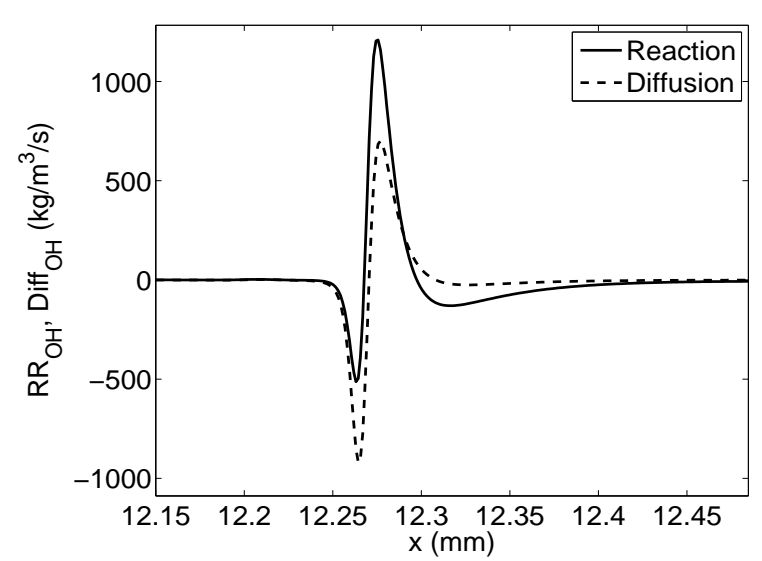

(a)

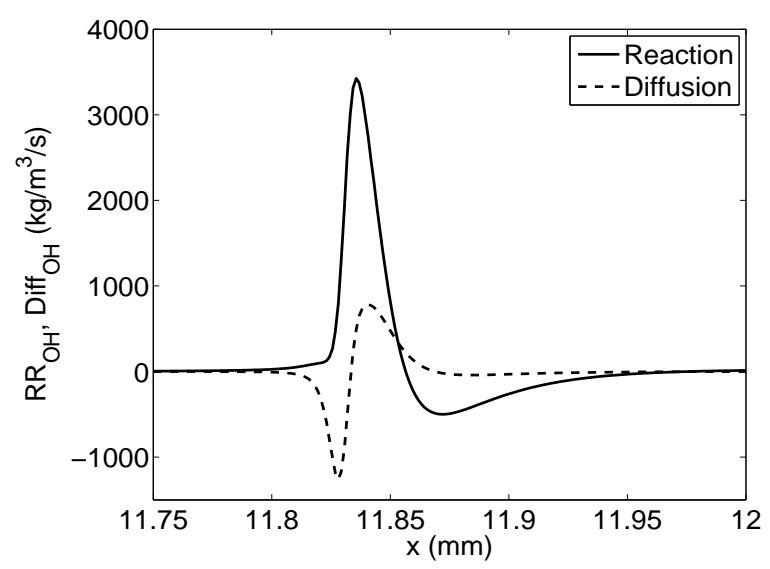

(b)

Figure 6: Reaction-diffusion balance for cases (a) 1D-a at $t=5.2 \mathrm{~ms}$ (b) $1 \mathrm{D}-\mathrm{b}$ at $t=6.5 \mathrm{~ms}$. Solid lines represent reaction rate $\left(R R_{O H}\right)$, dashed lines represent diffusion rate $\left(D i f f_{O H}\right)$. Domain boundaries are $(0,25) \mathrm{mm}$. The domain has been truncated to show only the region of interest.

$\mathrm{OH}$ radical, a key intermediate for both modes of combustion, as shown in Figure 6. The profile for case $1 \mathrm{D}$-a, shown at $t=5.2 \mathrm{~ms}$ in Figure $5(\mathrm{a})$, shows that diffusion and reaction are of comparable magnitude which is a key characteristic of deflagration flame-based combustion. The profile for case $1 \mathrm{D}-\mathrm{b}$, plotted at $t=6.5 \mathrm{~ms}$ in Figure $5(\mathrm{~b})$, shows reaction starting to dominate diffusion, a key characteristic of an ignition front. At $t=6.6 \mathrm{~ms}$ in Figure 5(b), the magnitude of the diffusion rate is almost negligible compared with that of the reaction rate (not shown here), a state corresponding to an ignition front. It should be noted that case 1D-a would also transition to an ignition front given a long enough domain and sufficient time for the upstream mixture to ignite.

To expand on the point above, an additional simulation, case 1D-c, was performed with uniform temperature, but with stratification in the n-heptane concentration. Stratification of the n-heptane concentration results in a gradient in ignition delay through composition stratification rather than temperature stratification. The form of the stratification was a 


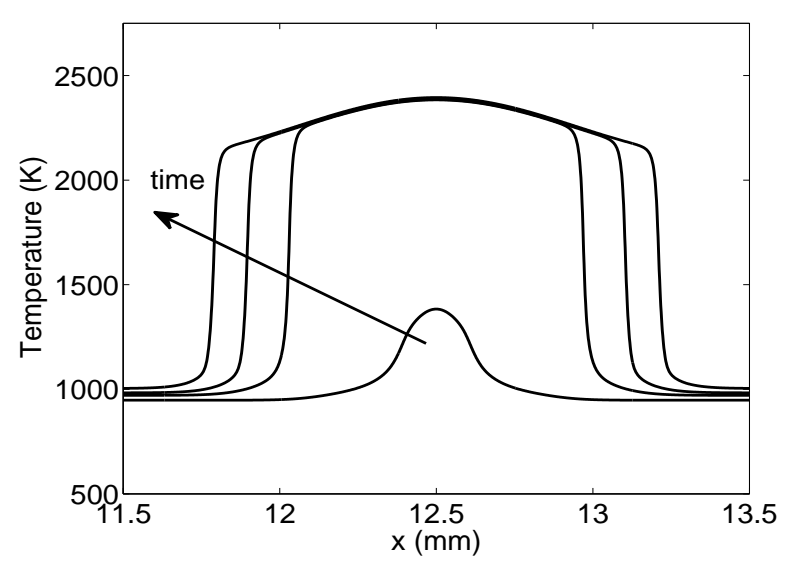

(a)

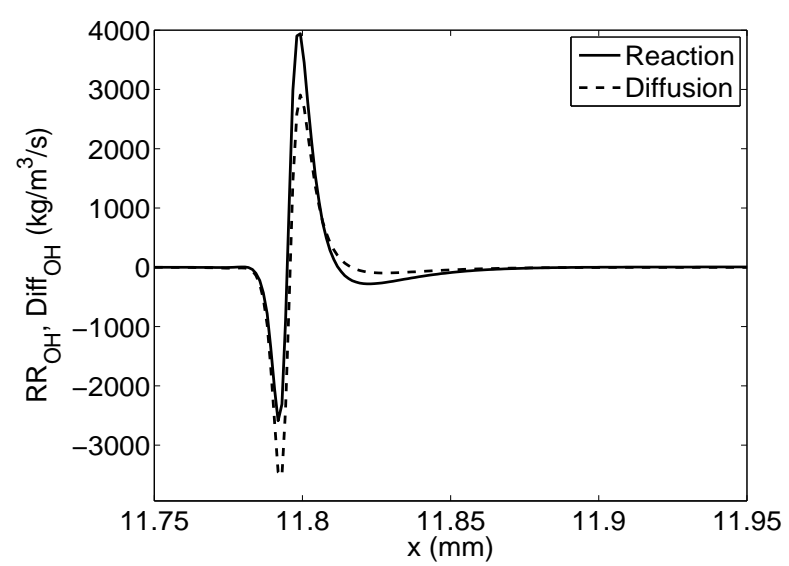

(b)

Figure 7: (a) Temperature profile (b) Reaction-diffusion balance for case 1D-c at $t=4.7 \mathrm{~ms}$ with uniform initial temperature. Solid lines represent reaction rate $\left(R R_{O H}\right)$, dashed lines represent diffusion rate $\left(D i f f_{O H}\right)$. The domain boundaries are $(0,0.025) \mathrm{m}$. The domain has been truncated to show only the region of interest.

Gaussian with the same width as the temperature stratification in the previous two cases. Figure 7 shows the temperature profile at several times and the reaction and diffusion rates at $\mathrm{t}=4.7 \mathrm{~ms}$ for case 1D-c. The results show that in this case, the dominant mode of combustion is flame propagation. It is remarkable that ignition kernels propagate as premixed flames with stratification in fuel concentration alone in the absence of thermal stratification at $t=0$. This behavior is also observed in the two dimensional simulations presented in the following section.

\section{Two dimensional simulations}

To further explain the trends observed in the one-dimensional simulations, two-dimensional simulations with zero-mean, random velocity fluctuations and stratification in composition and/or temperature were performed. The length scale of the velocity fluctuations was chosen to be comparable to the length scale of the temperature and n-heptane concentration 


\begin{tabular}{|c|c|c|c|c|c|c|c|c|}
\hline \hline Case \# & $\bar{T}$ \\
$(K)$ & $\begin{array}{c}T_{r m s} \\
(K)\end{array}$ & $\begin{array}{c}l_{T} \\
(m m)\end{array}$ & $\bar{\phi}_{n \text {-heptane }}$ & $\phi_{\text {rms,n-heptane }}$ & $\begin{array}{c}l_{\phi} \\
(m m)\end{array}$ & $\bar{\phi}_{\text {overall }}$ & $\begin{array}{c}\text { Pressure } \\
\text { profile }\end{array}$ \\
\hline \hline 2D-a & 714 & 15 & 1.2 & 0.44 & 0.04 & 1.2 & 0.69 & Senkin \\
\hline 2D-b & 714 & 15 & 1.2 & 0.23 & 0.04 & 1.2 & 0.52 & Senkin \\
\hline 2D-c & 714 & 15 & 1.2 & 0.44 & 0.04 & 1.2 & 0.69 & Expt \\
\hline 2D-d & 714 & 15 & 1.2 & 0.23 & 0.04 & 1.2 & 0.52 & Expt \\
\hline 2D-e & 714 & 0 & - & 0.44 & 0.04 & 1.2 & 0.69 & Expt \\
\hline 2D-f & 714 & 0 & - & 0.23 & 0.04 & 1.2 & 0.52 & Expt \\
\hline
\end{tabular}

Table 2: Physical parameters for the two dimensional simulations. All simulations had a nearly constant background equivalence ratio with respect to iso-octane, $\bar{\phi}_{\text {iso-octane }}=0.27$ and $\phi_{\text {rms,iso-octane }} \approx 0$. The magnitude of velocity fluctuations was $u_{r m s}=0.52 \mathrm{~m} / \mathrm{s}$ with the integral length scale based on the twopoint velocity correlation, $l_{11}=0.7 \mathrm{~mm}$. "Senkin" denotes that the mean pressure was obtained simply by substituting the time variable into the SENKIN expression for pressure trace with the initial conditions from Table 3. "Expt" denotes that the mean pressure was constrained to follow the experimental pressure trace in [7] using the model given by Eq. 14.

\begin{tabular}{|c|c|}
\hline \hline $\bar{T}_{0}(\mathrm{~K})$ & 714 \\
\hline $\bar{P}_{0}$ (bar) & 17.4 \\
\hline Engine RPM & 1200 \\
\hline $\begin{array}{c}\text { Initial crank angle } \\
\left({ }^{\circ} B T D C\right)\end{array}$ & 20 \\
\hline $\begin{array}{c}\text { Compression ratio } \\
\text { Comp }\end{array}$ & 10.7 \\
\hline
\end{tabular}

Table 3: Mean initial conditions for isentropic compression for the 2-D simulations (Cases 2D-a through 2D-f). 
fluctuations and the time scale of the velocity fluctuations was chosen to be comparable to the homogeneous ignition delay time. Note that, since two-dimensional turbulence is not representative of the three-dimensional flow field, analysis of turbulence-chemistry interactions must be performed with caution. However, two-dimensional simulations are useful to provide trends with respect to the change in output quantities of interest for a given change in input parameters in the presence of the mixing influence of the imposed velocity fluctuations. Table 2 lists the parameters for all of the cases simulated in the present study. Cases 2D-a and 2D-b use the "Senkin" method for emulating pressure as described in [22] whereas Cases 2D-c through 2D-f employ the pressure imposition technique described in Section 2 above. The pressure profile, denoted by "Expt" for these cases, uses the pressure trace from an RCCI engine experiment described in [7]. This experiment was also performed with iso-octane and n-heptane and involves a parametric sweep in injection timing for the high-reactivity fuel, n-heptane. Cases 2D-a, 2D-c and 2D-e have the same (higher) mean equivalence ratio with respect to n-heptane, whereas cases 2D-b, 2D-d and 2D-f have a lower equivalence ratio with respect to n-heptane. The equivalence ratio with respect to iso-octane was the same for all six cases.

\subsection{Initial and boundary conditions}

The domain size for all two-dimensional cases is $L_{x}=4.0 \mathrm{~mm}$ and $L_{y}=4.0 \mathrm{~mm}$ with periodic boundary conditions applied in both directions. To ensure reliable statistics with the periodic boundary conditions, the domain size was chosen to ensure the existence of at least 5 integral length scales in each direction. Another reason for this choice is to ensure a turbulence time scale, $\tau_{\text {turb }}=l_{11} / u_{r m s}$ that is comparable to a real engine even though the velocity scale and Reynolds numbers cannot be matched [23]. The simulations for all cases in Table 2 were initialized with random velocity fluctuations with zero mean velocity through a Passot-Pouquet spectrum, such that $u_{r m s}=0.52 \mathrm{~m} / \mathrm{s}$ and the integral length 


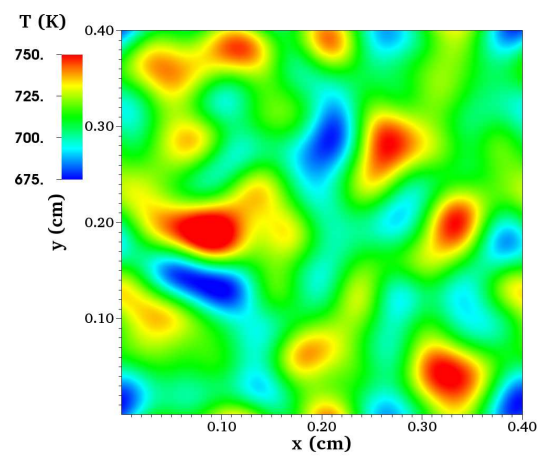

(a)

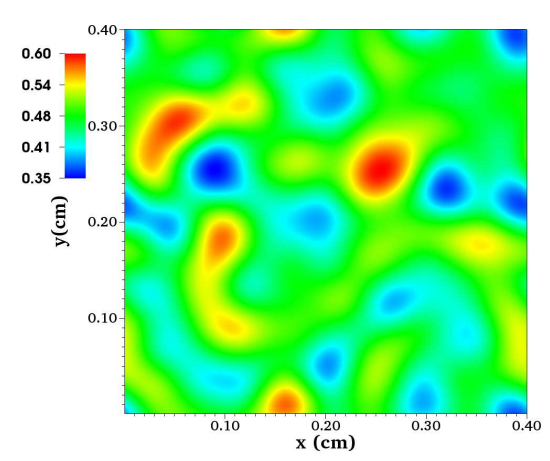

(b)

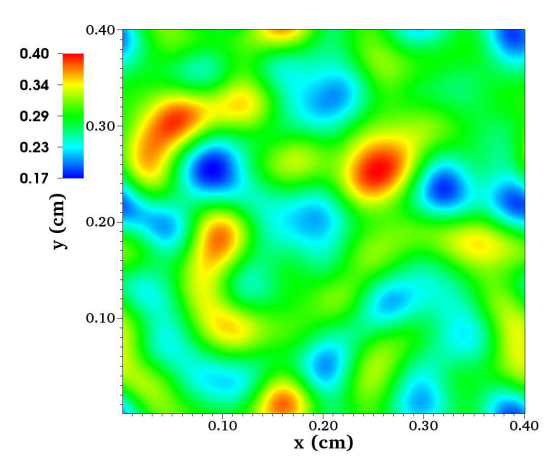

(c)

Figure 8: Initial conditions for (a) Temperature for cases 2D-a through 2D-d (b) $\phi_{n-h e p t a n e}$ for cases 2D-a, $2 \mathrm{D}-\mathrm{c}, 2 \mathrm{D}-\mathrm{e}$ (c) $\phi_{n-\text { heptane }}$ for cases 2D-b, 2D-d, 2D-f.

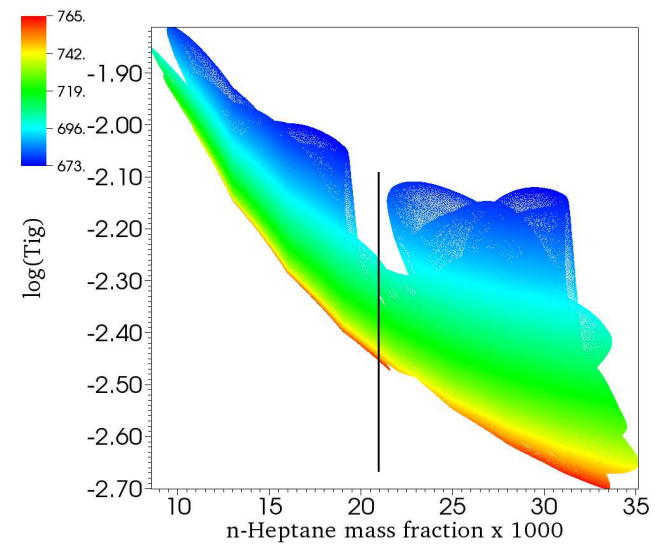

Figure 9: Combined scatter plot of the (base-10) log of 0-D ignition delay times versus n-heptane mass fraction at $t=0$ for cases $2 \mathrm{D}-\mathrm{c}$ and $2 \mathrm{D}$-d colored by temperature. Points to the left of the solid vertical line belong to $2 \mathrm{D}-\mathrm{d}$, points to the right belong to $2 \mathrm{D}-\mathrm{c}$. 
scale, $l_{11}=0.7 \mathrm{~mm}$. The temperature field for cases $2 \mathrm{D}$-a through $2 \mathrm{D}$-d was also initialized with the same spectrum providing a consistent mean temperature, $\bar{T}=714 K$, and variance, $T_{r m s}=15 \mathrm{~K}$, with a fluctuation length scale, $l_{T}=1.2 \mathrm{~mm}$. It has been shown that very large initial gradients in temperature favor flame propagation for all fuels $[17,24]$, whereas in this study we are interested in premixed flame fronts generated through reaction and mixing. Hence this value of $l_{T}$ was chosen to minimize peak initial gradients in temperature while ensuring enough samples for statistics. A uniform initial temperature field was specified for cases 2D-e and 2D-f to isolate the effect of fuel reactivity stratification on the competition between flame propagation and ignition fronts. Reactivity is interpreted here in terms of the fuel blend at any given location. Since the fuel in this study is a blend of n-heptane and iso-octane, a higher fraction of n-heptane compared to iso-octane at any given location implies greater reactivity. Figure 8(a) shows the temperature field at $t=0$.

In dual fuel RCCI engines, the low reactivity fuel (e.g., iso-octane) is typically premixed and the high reactivity fuel (e.g., n-heptane) is direct-injected. Thus, the equivalence ratio of iso-octane is nearly uniform throughout the combustion chamber. The direct-injected n-heptane would then impose a distribution of equivalence ratio, temperature, and local octane number (i.e., PRF number) onto the uniform iso-octane distribution (see Figure 2). The stratification of the direct-injected, low reactivity fuel (e.g., n-heptane) can be adjusted by adjusting the SOI timing [14]. In the present study, the concentration of iso-octane was held constant at $\phi_{\text {iso-octane }}=0.27$. The DNS considers only a $4 \mathrm{~mm}$ by $4 \mathrm{~mm}$ two-dimensional slice of the combustion chamber. Thus, it is not possible to consider the full distribution of the direct-injected fuel; however, the effect of equivalence ratio, temperature, and local octane number stratification can be evaluated by imposing a fluctuating concentration field of n-heptane. Two levels of mean n-heptane concentration were simulated as shown in Figures $8(\mathrm{~b})$ and $8(\mathrm{c})$, such that the equivalence ratio with respect to $\mathrm{n}$-heptane alone, $\phi_{n-\text { heptane }}$ 
varied from 0.35 to 0.6 in one case and from 0.17 to 0.4 in the other. This can be thought of as equivalent to simulating two different locations in Figure 2. It must be noted that the purpose of the present investigation is not to reproduce the exact conditions from a specific set of engine experiments, but rather to explain the relative roles of flame propagation and ignition fronts and its sensitivity to fuel reactivity stratification induced by direct injection.

Another way to understand the initial thermochemical space is through the homogeneous ignition delay described in Section 3.1. Figure 9 shows the combined scatter of the logarithm of the ignition delay with respect to the mass fraction of n-heptane for both cases $2 \mathrm{D}$-c and 2D-d colored by the initial temperature. Since the ranges of n-heptane concentrations for the two cases are adjacent to each other with very little overlap, the overall plot appears as a continuum of states. Nevertheless, a solid vertical line has been added to demarcate the separation point on the x-axis. Points to the left of the solid vertical line belong to $2 \mathrm{D}-\mathrm{d}$, points to the right belong to $2 \mathrm{D}$-c. Since cases $2 \mathrm{D}-\mathrm{a}$ and $2 \mathrm{D}-\mathrm{b}$ have the same initial conditions as cases 2D-c and 2D-d respectively, the same plot applies to them as well. The observation regarding the sensitivity of $\tau_{i g}$ with respect to n-heptane concentration made earlier with respect to Figure 4 is quite evident in this plot. In fact, the variation in $\tau_{i g}$ for case 2D-d (lower concentration of n-heptane) due to concentration alone is greater than the variation due to temperature stratification.

All cases used inert mass source terms to emulate the pressure change due to piston compression. Cases 2D-a and 2D-b used the formulation described in Bhagatwala et al. [22], which adds or subtracts mass source terms uniformly based on the zero-dimensional SENKIN expression [9]. One of the disadvantages of using this method of emulating pressure change is that the pressure feedback from combustion due to the small DNS domain size is over predicted, giving much higher pressures than observed in real engines. Since autoignition 


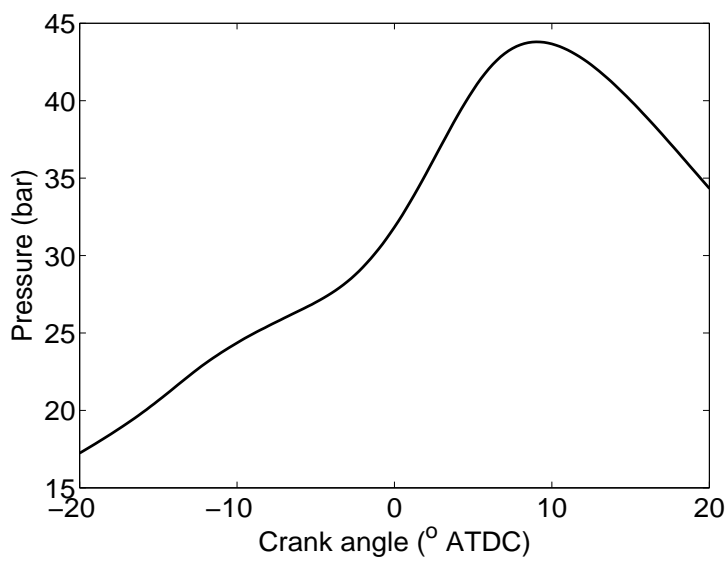

(a)

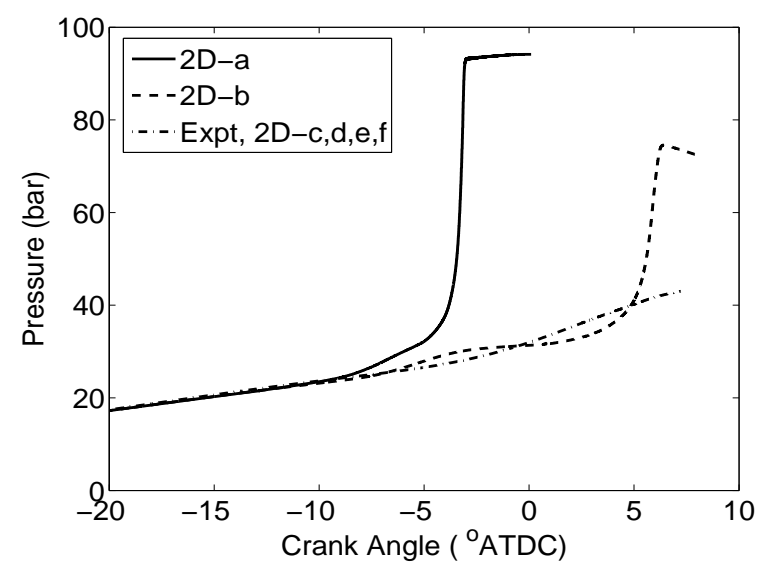

(b)

Figure 10: (a) Pressure trace from the RCCI experiment by Kokjohn [7] used for cases 2D-c to 2D-f, and (b) experimental pressure trace in (a) overlaid with pressure traces from cases 2D-a and 2D-b. The dash-dotted line in (b) is the same as the solid line in (a) but plotted on a different scale. Note that the pressure trace denoted by the dash-dotted line marked "Expt" is identical to that recovered by the DNS cases 2D-c,d,e,f.

is strongly dependent on pressure, this method is likely to favor ignition fronts over flame propagation. To overcome this limitation, the alternative method of imposing the experimental pressure trace described above was implemented for cases 2D-c through 2D-f.

\subsection{Time traces}

Figure 11 shows traces of domain averaged heat release rate and peak temperature for all six cases highlighting several trends. The ignition delay associated with the first stage, corresponding to low-temperature heat release (LTHR) is nearly the same for all cases. Ignition delay is taken to be the time at which the magnitude of mean temperature gradient is maximum, with $t=0$ being the start of the simulation.. Predictably, the ignition delay for the high temperature, second-stage heat release is shorter for the cases with higher nheptane concentration (2D-a, 2D-c and 2D-e). Further, this delay is shorter for case 2D-a compared to cases 2D-c and 2D-e due to the higher pressure during the high-temperature 


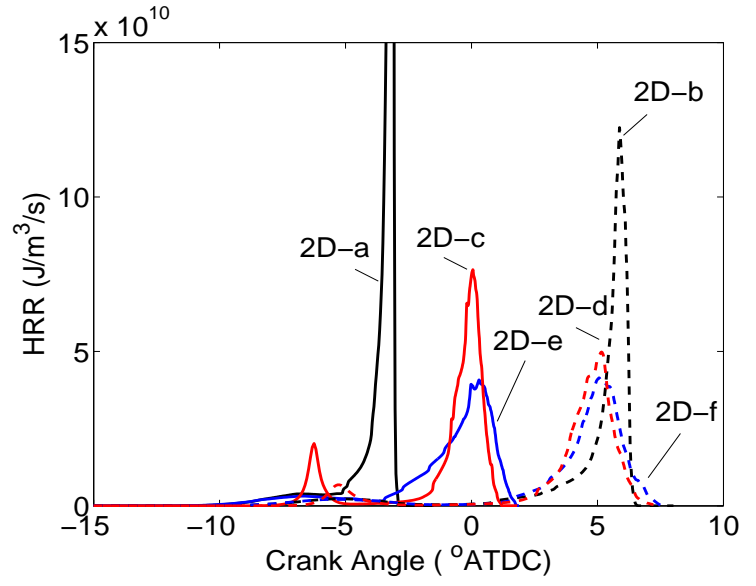

(a)

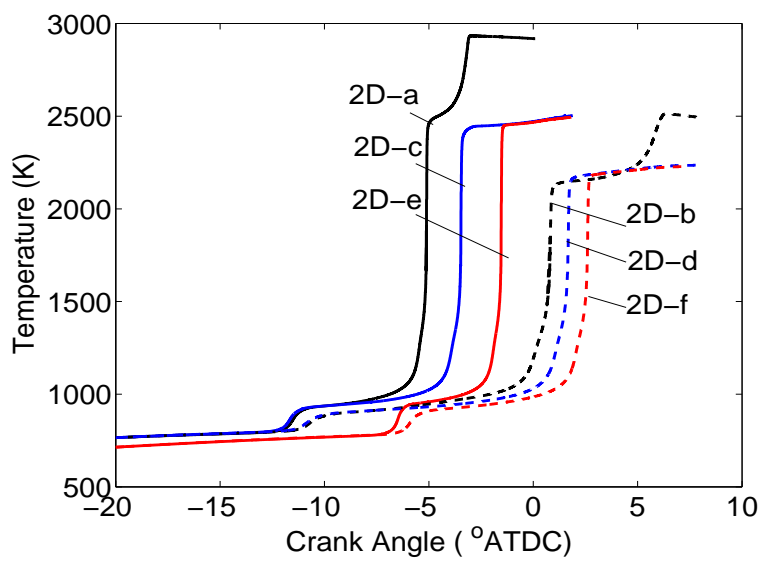

(b)

Figure 11: Traces of (a) average heat release rate (HRR), and (b) peak temperature for all six cases. Solid lines denote cases with higher overall equivalence ratio $(\phi=0.69)$, dashed lines denote cases with lower equivalence ratio $(\phi=0.52)$

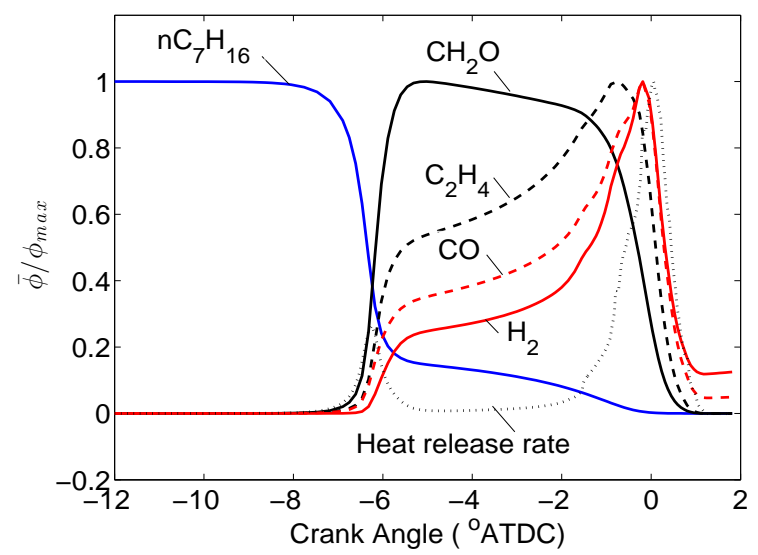

Figure 12: Temporal evolution of normalized species mass fractions and heat release rate for case 2D-e. The overbar (.) denotes an average over the entire simulation domain. The dotted line represents heat release rate. 
ignition event, as observed in Figure 10(b). However, this effect is not seen for case 2D-b, for which the peak HRR associated with high-temperature ignition is slightly delayed compared to cases 2D-d and 2D-f, in spite of the pressure being much higher. To understand this, note that the peak heat release for these three cases occurs after top dead center (TDC), i.e. during the expansion stroke when the mean pressure is decreasing. This retards the time of peak HRR for all three cases where it occurs after TDC (2D-b, 2D-d, 2D-f). The pressure for case $2 \mathrm{D}-\mathrm{b}$ is higher than for the other two cases between -5 and 0 crank angle degrees and lower between 0 and 5 degrees. It rises sharply only after 5 degrees, which is approximately the point of peak HRR. Hence, the observed behavior is consistent with the pressure trace between -5 and 5 degrees. Note also that higher pressures are associated with higher peak HRR values, a consequence of the fact that higher pressure favors ignition fronts over flame propagation. This will be discussed in more detail in the next section. These results further underscore the shortcomings of using the Senkin approach, which mispredicts both the timing and magnitude of the heat release and also the mode of combustion, as will be seen in later sections. It should also be noted that the Senkin approach, while not as good as the one based on the experimental pressure trace, would still yield results that are closer to the experiment than a constant volume approach. The temperature profiles in Figure 11(b) show higher peak temperatures for cases with the higher overall equivalence ratio, as expected. The peak temperature profiles show the temperature of the hottest parcel of fluid at any given time.

Figure 12 shows the temporal evolution of normalized domain average species concentrations for case 2D-e. It can be seen in Figure 12, that after the LTHR stage, the original n-heptane is almost entirely consumed, most of it having decomposed to $\mathrm{CH}_{2} \mathrm{O}, \mathrm{C}_{2} \mathrm{H}_{4}$ and other smaller molecules. It is the oxidation of these smaller hydrocarbons (as well as $H_{2}$ and CO) that drives the high temperature heat release (HTHR) [36]. 


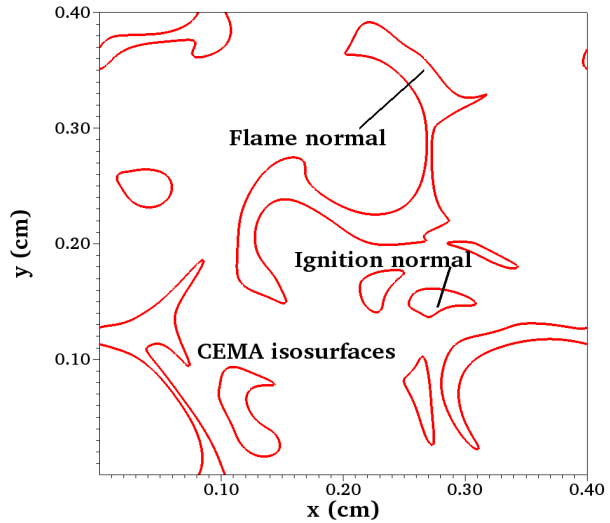

(a)

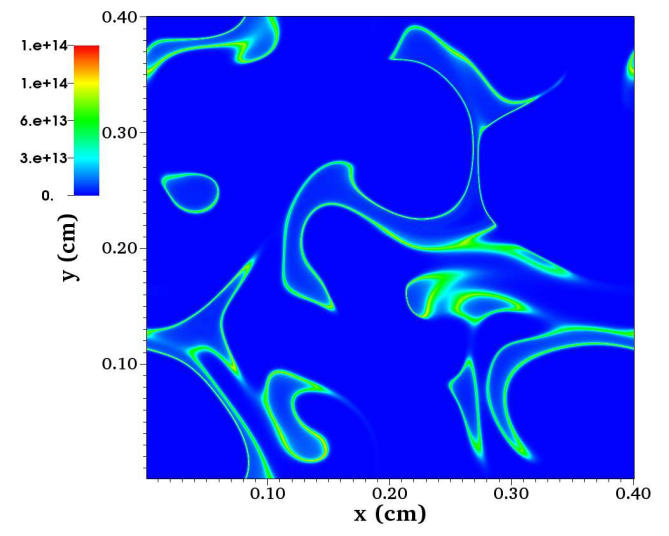

(b)

Figure 13: (a) Schematic showing selection of CEMA isosurfaces and selected normals along them, and (b) filled contour plot of heat release rate for case $2 \mathrm{D}$-a.

\subsection{Combustion mode analysis}

In this section, the two-dimensional simulations are analyzed to quantify the extent of flame propagation versus ignition fronts. We identify the instantaneous location of the ignition front, measure its propagation speed and also study the properties along the local normal to the ignition front. The extent of flame propagation versus ignition fronts is quantified for the two-dimensional simulations through analyzing the reaction diffusion balance along the local normals on the ignition front. To do so, first, a good definition of the ignition front is required. Note that the method must be robust enough to reliably flag both an autoigniting element as well as one burning through deflagration. In our earlier DNS studies [16, 17] we used the iso-contour of the fuel mass fraction to denote the location of the ignition front. However, in the presence of mixture composition stratification, a single value of mass fraction that could define the iso-contour does not exist as seen from figure 12. Furthermore, there is significant consumption of reactants during low temperature heat release in the current cases, which does not exist in the $H_{2}$ fuel used in the previous studies. Therefore, a different 


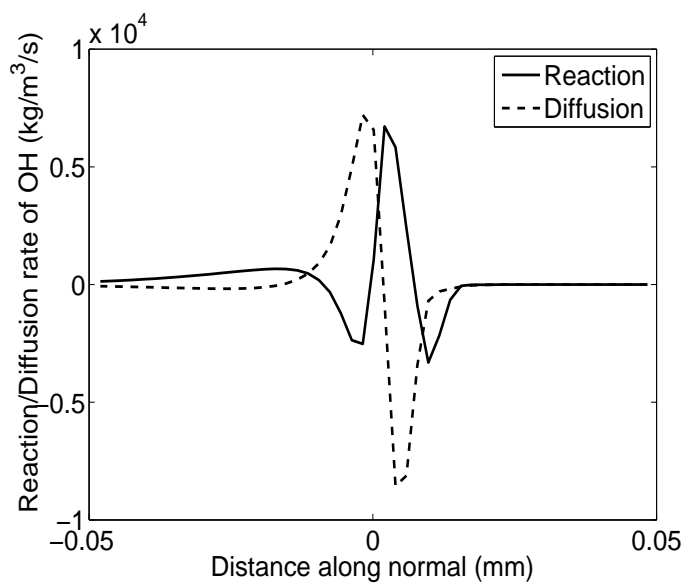

(a)

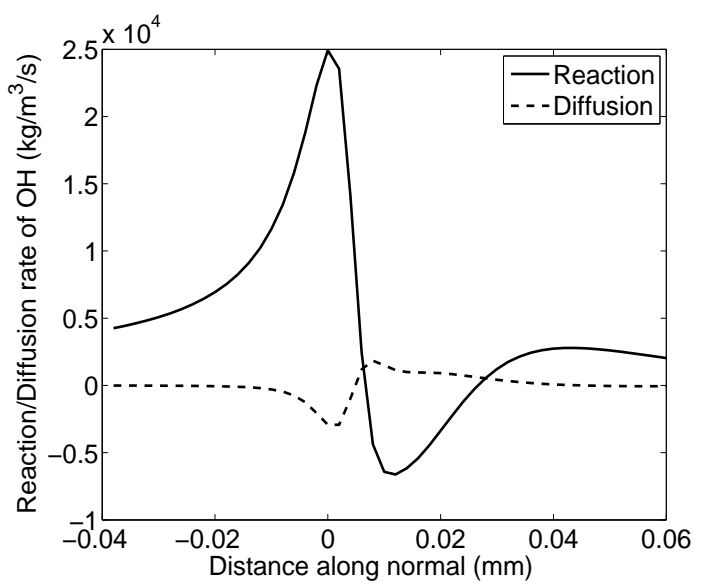

(b)

Figure 14: Reaction-diffusion balance along representative normals for (a) flame propagation, and (b) an ignition front for case 2D-a. The origin on the $\mathrm{x}$-axis represents the location of the flame/ignition front as identified by the CEMA analysis.

diagnostic is needed to identify the location of the ignition front.

In the present study, the chemical explosive mode analysis (CEMA) [20, 21], which identifies both flames and ignition fronts, based on instantaneous conditions is used as the diagnostic criterion. The CEMA methodology has been described in detail in several publications $[20,21,22,24]$; thus, only a brief description is provided here. It involves computing the eigenvalues of the Jacobian formed by differentiating the reaction rate vector with respect to the vector of the species mass fractions. The leading eigenvalue of this Jacobian, $\lambda_{e}$, is termed the chemical explosive mode (CEM) for the mixture at any point locally. $\lambda_{e}$ is thus a function of space and time, $\lambda_{e} \equiv \lambda_{e}(\mathbf{x}, t)$. The property of $\lambda_{e}$ that will be used here is that it is positive in an unburned fuel-air mixture that is about to ignite, negative in the burnt region and has a zero crossing on the burning surface itself. Thus, the surface $\lambda_{e}(\mathbf{x})=0$ represents the burning surface at any point in time. While it is possible to estimate the 
propagation mode (flame or ignition front) using the magnitude of the upstream $\lambda_{e}$ value, this approach will not be discussed here since the first principles-based procedure followed in this paper is more reliable and accurate. The reader is referred to an earlier publication [22] for details on the CEMA-based approach. Figure 13(a) shows the burning surface for case 2D-a at $t=2.34 m s$, near the point of peak HRR. A comparison with Figure 13(b) which shows the heat release at the same time, shows that both ignition fronts (thick zones) and flame propagation (thin rings) are present and are faithfully represented by the CEMA isosurface, $\lambda_{e}(\mathbf{x})=0$.

The isocontour of $\lambda_{e}=0$ is then extracted from the DNS data through triangulation using the marching cubes algorithm [32]. At each vertex of the isocontour, a local normal to the surface is obtained using the local gradient of $\lambda_{e}$, which defines the isocontour. If the local gradient is $\nabla \lambda_{e}$, then the unit normal vector is given by $\hat{n}=\nabla \lambda_{e} /\left|\nabla \lambda_{e}\right|$. The normal vector extends on both sides of the surface and it is terminated using a distance function. The distance function, $d$, is defined at every point in the domain as the distance to the nearest isocontour surface. Where the distance function does not increase monotonically along the normal, indicates the point where the normal is closer to a different vertex on the isosurface and hence terminates the normal. This procedure makes the normal line segments truly representative of the vertex from which they originated.

Also shown in Figure 13(a) are representative normals to deflagration and ignition front isosurfaces. Figure 14 shows the diffusion and reaction rates of $O H$ interpolated on to those normals. It can be seen that, for the flame (deflagration), both are in balance, though not spatially collocated. Hence the Damköhler number as defined by Eq. 15, is of order unity, $D a \sim O(1)$. For the ignition front normal, the reaction rate is much greater than the diffusion rate, and hence $D a \gg 1$. As shown in Figure 14, the extrema for reaction rate 


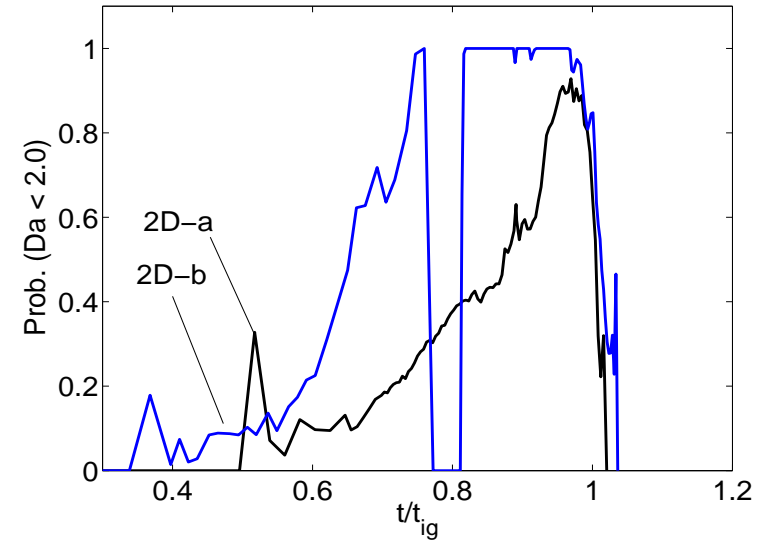

(a)

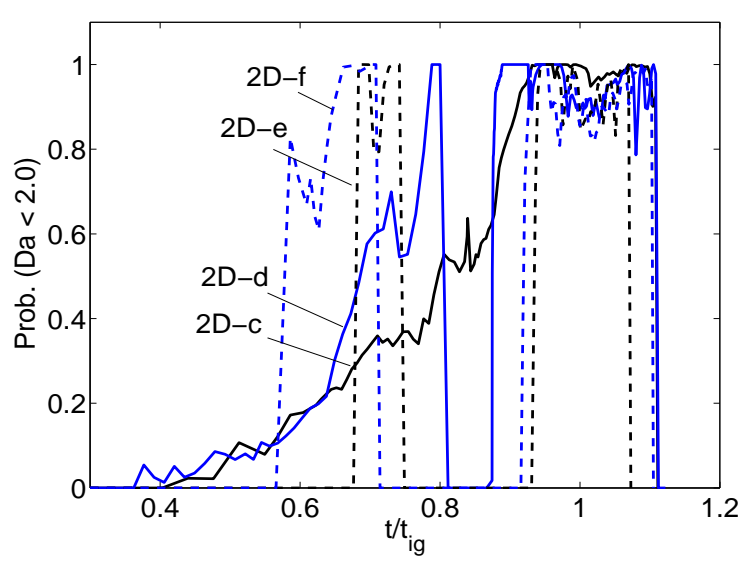

(b)

Figure 15: Evolution of the fraction of points igniting via the deflagration mode for cases (a) 2D-a, 2D-b, and (b) 2D-c, 2D-d, 2D-e, 2D-f. Cases in (a) use the SENKIN method for emulating pressure whereas cases in (b) use the experimental pressure trace. Solid lines indicate cases with both mixture and thermal stratification at $t=0$. Dashed lines indicate cases with only mixture stratification and uniform temperature at $t=0$. The color black represents cases with higher overall equivalence ratio $(\phi=0.69)$, whereas blue represents cases with lower overall equivalence ratio $(\phi=0.52)$.

and diffusion flux are generally not collocated in a flame, and hence a simple ratio of these quantities at a given spatial location is erroneous as a metric to characterize the combustion mode of a burning element. Therefore, it is important to categorize the combustion mode by analyzing the reaction diffusion balance along the local normal to the propagating front.

A Damköhler number, Da, for each flame or autoigniting surface element is computed, defined as the ratio of the maximum magnitude of the reaction rate to the maximum magnitude of the diffusion rate of $O H$, along a given normal to the burning surface,

$$
D a_{O H}=\frac{\max \left|\omega_{O H}\right|}{\max \left|-\nabla \cdot\left(\rho D_{O H} \nabla Y_{O H}\right)\right|} .
$$

Figure 15 shows the fraction of the isosurface normals with $D a$ as defined by Eq. 15, having 
a value less that 2.0. This is taken as the fraction of combustion heat release occurring via the flame propagation mode. The profiles have been normalized by the ignition delay time $\left(t_{i g}\right)$ for each case, where $t_{i g}$ is taken to be the time at which $50 \%$ of the total heat release has occurred. This roughly corresponds to the peak of the heat release rate profile shown in Figure 11(a). It can be seen that low temperature heat release (LTHR), which is dominant between $0.4\left(t / t_{i g}\right)$ to $0.8\left(t / t_{i g}\right)$, occurs via both flame propagation and through ignition fronts. Interestingly, the two cases with uniform initial temperature (2De and 2D-f) show a shorter duration of LTHR and almost all of it through flame propagation.

It is also evident that for high temperature heat release (HTHR), which largely occurs between $0.8\left(t / t_{i g}\right)$ to $1.1\left(t / t_{i g}\right)$, higher pressure during this phase in cases $2 \mathrm{D}$-a and $2 \mathrm{D}-\mathrm{b}$ (i.e., the two cases with the SENKIN imposed pressure profiles) results in a slightly greater proportion of ignition front propagation. This is especially true during the final stages, after $1.0\left(t / t_{i g}\right)$, where the cases with the experimental pressure trace show burning occurring through flame propagation until all of the fuel is consumed. Among the cases with the experimental pressure profile, cases with temperature stratification (2D-c and 2D-d) have a slightly higher fraction of flame propagation during HTHR than the corresponding cases with uniform temperature (2D-e and 2D-f). This is consistent with the expectation that higher temperature stratification favors flame propagation. However, it is remarkable that cases with a uniform initial temperature still result in combustion occurring primarily via flame propagation rather than ignition fronts. This characteristic seems intrinsic to fuels with high reactivity, such as n-heptane and in contrast to low-reactivity fuels such as pure iso-octane or ethanol which ignite almost exclusively via ignition fronts for the same level of stratification. At a large enough magnitude of stratification (fuel or temperature), almost all fuels would display some level of flame propagation [17, 24]. It is worth noting that a zero probability of $D a<2.0$ in Figure 15 may also indicate a lack of combustion activity 


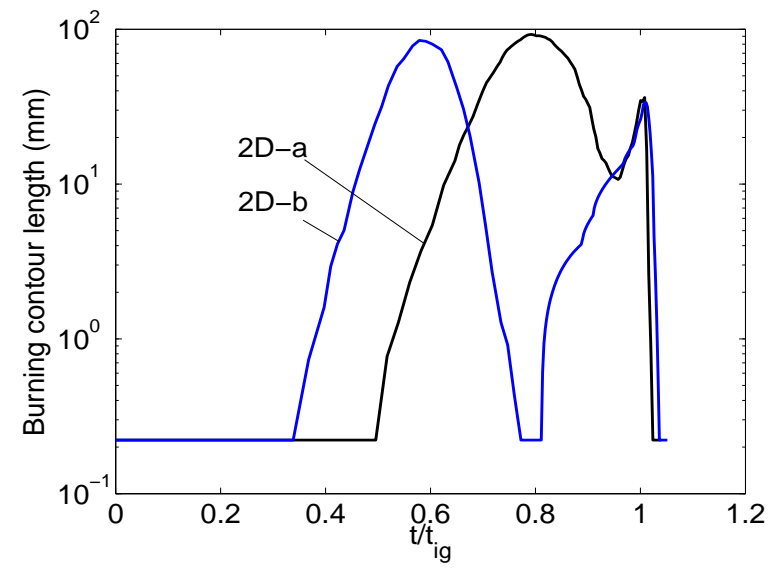

(a)

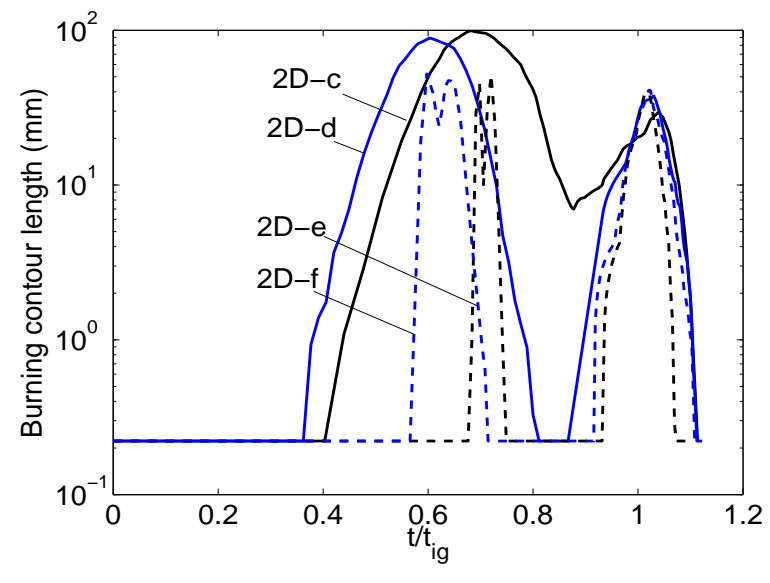

(b)

Figure 16: Evolution of the total length of the burning contour for cases (a) 2D-a, 2D-b, and (b) 2D-c, 2D-d, 2D-e, 2D-f. Cases in (a) use the SENKIN method for emulating pressure whereas cases in (b) use the experimental pressure trace. Solid lines indicate cases with both mixture and thermal stratification at $t=0$. Dashed lines indicate cases with only mixture stratification and uniform temperature at $t=0$. The color black represents cases with higher overall equivalence ratio $(\phi=0.69)$, whereas blue represents cases with lower overall equivalence ratio $(\phi=0.52)$.

(LTHR or HTHR) in the simulation domain. This is illustrated in Figure 16 which shows the aggregate length of the $\lambda_{e}=0$ contour as a function of time. Cases 2D-a and 2D-c show a continuous transition from LTHR to HTHR, whereas all other cases have a clear separation between the two. To provide further confidence in this methodology, the Damköhler number computation was repeated by substituting other important radical species such as $\mathrm{HO}_{2}$ and $\mathrm{HCO}$ in place of $\mathrm{OH}$ in Eq. 15, and the results were found to be very similar to those with $\mathrm{OH}$.

To further quantify the role of ignition fronts and flame propagation, a second technique was used. This involved computing the consumption speed $s_{c}$, defined as

$$
s_{c}=-\frac{1}{\rho_{u} Y_{F, u}} \int_{u}^{b} \dot{\omega}_{F} d n
$$




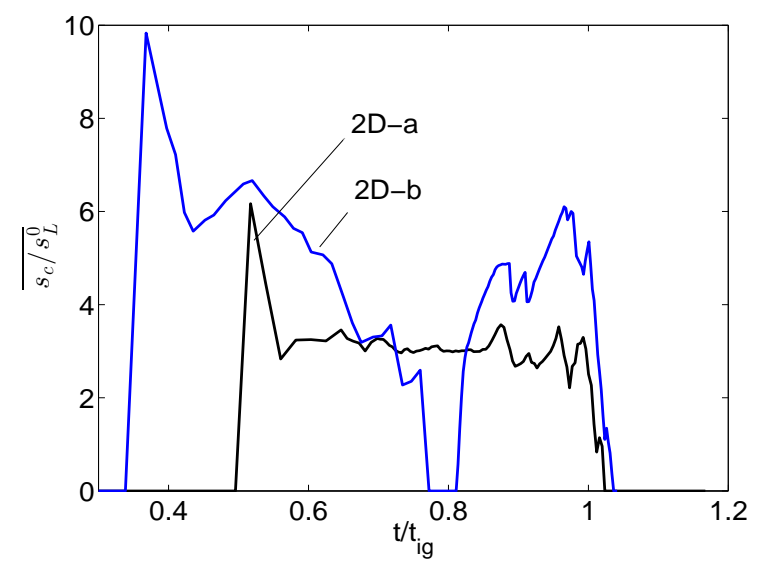

(a)

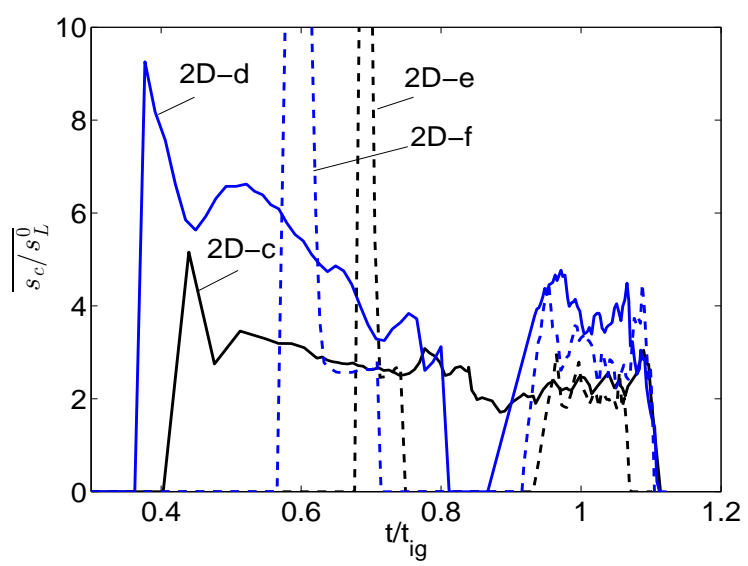

(b)

Figure 17: Evolution of area-weighted mean consumption speed for cases (a) 2D-a, 2D-b, and (b) 2D-c, 2D-d, 2D-e, 2D-f. Cases in (a) use the SENKIN method for emulating pressure whereas cases in (b) use the experimental pressure trace. Solid lines indicate cases with both mixture and thermal stratification at $t=0$. Dashed lines indicate cases with only mixture stratification and uniform temperature at $t=0$. The color black represents cases with higher overall equivalence ratio $(\phi=0.69)$, whereas blue represents cases with lower overall equivalence ratio $(\phi=0.52)$.

where the subscript $u$ refers to the unburnt state upstream of the flame and $b$ refers to the burnt state downstream. The fuel mass fraction and reaction rate are taken as $Y_{F, u}=Y_{n-h e p t a n e, u}+Y_{i s o-o c t a n e, u}$ and $\dot{\omega}_{F}=\dot{\omega}_{n-\text { heptane }}+\dot{\omega}_{\text {iso-octane }}$, respectively. The integral is computed using Simpson's 3/8 rule along the flame normal. The consumption speed is normalized with the unstrained laminar flame speed, $s_{L}^{0}$, for a comparable PRF mixture, at the given unburnt equivalence ratio, temperature and pressure. The method for computing $s_{L}^{0}$ is detailed in the Appendix. The motivation for this normalization is that the magnitudes of $s_{c} / s_{L}^{0}$ of order unity imply flame propagation, whereas $s_{c} / s_{L}^{0} \gg 1$ indicate predominantly ignition fronts. Multiple states between these two limits are possible, and they indicate the relative proportion of the two modes of propagation.

Figure 17 plots the area-weighted mean consumption speed for all six cases. This quantity 
can also be thought of as a normalized burning rate since total heat release at any point

in time can be written as $\Sigma \int_{A} s_{c} d A$, where the summation is carried out over all burning elements in the domain. Higher consumption speeds for cases 2D-b and 2D-d (higher proportion of iso-octane compared to n-heptane) during LTHR, compared with 2D-a and 2D-c (higher proportion of n-heptane compared to iso-octane) indicate a greater fraction of ignition front propagation in mixtures with a higher octane number. A similar trend is seen during HTHR for those cases as well. Cases 2D-e and 2D-f (uniform initial temperature) show a greater fraction of ignition front propagation at the beginning of LTHR, but switch to deflagration during the latter half of LTHR. Combustion during HTHR for both cases appears to be dominated by deflagration, though more so for case 2D-e due to its lower octane number and higher overall equivalence ratio. These results are consistent with those of Tamagna et al [33], who also report a greater degree of flame propagation with increasing concentration of the high-reactivity fuel.

Figure 18 shows a PDF of the consumption speed at $t=t_{i g}$, recalling that $t_{i g}$ is the time at which $50 \%$ of the total heat release has occurred. The profiles for cases $2 \mathrm{D}$-a, 2D-c and 2D-e show strong peaks between $1<s_{c} / s_{L}^{0}<5$, corresponding to the deflagration mode. Cases 2D-b, 2D-d and 2D-f on the other hand, show strong peaks between $5<s_{c} / s_{L}^{0}<15$, indicating greater propensity for generating ignition fronts. These plots support the finding that higher n-heptane concentration favors deflagration and lower n-heptane concentration favors ignition fronts.

\subsection{Analysis of the ignition delay gradient}

Zeldovich [34] has proposed that the mode of combustion propagation in a premixed system depends on the initial gradient in the homogeneous (0-D) ignition delay encountered in the 


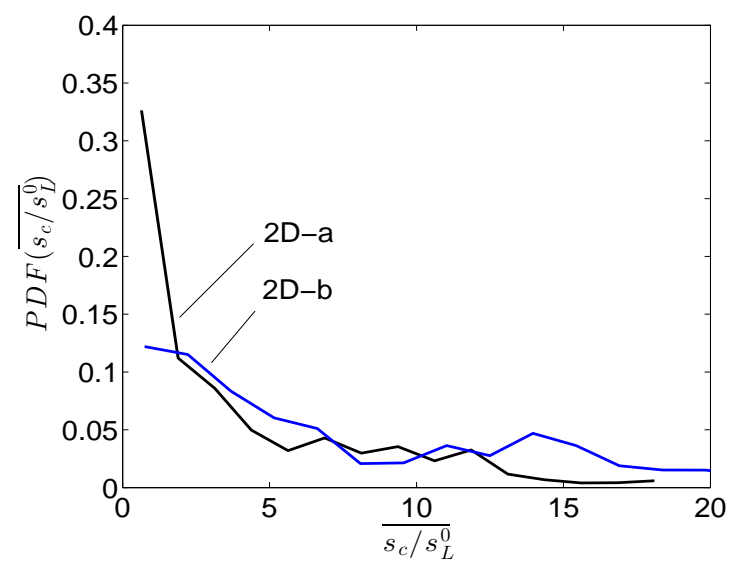

(a)

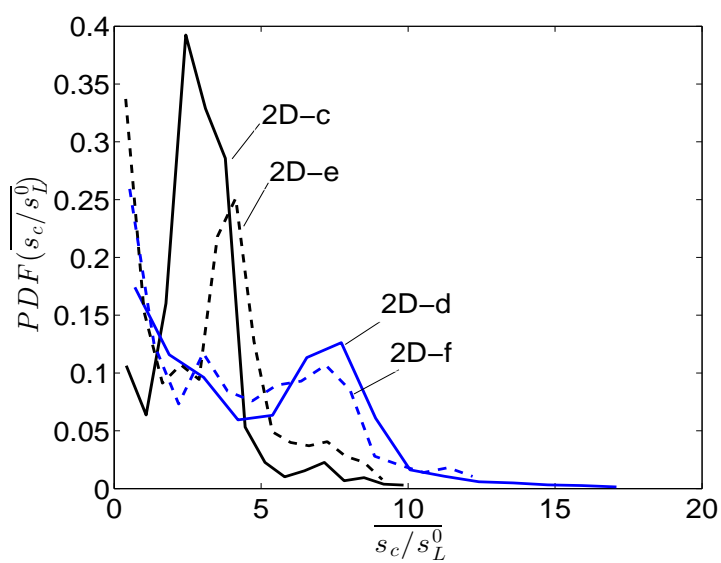

(b)

Figure 18: PDF of consumption speed at $t=t_{i g}$ for cases (a) 2D-a, 2D-b, and (b) 2D-c, 2D-d, 2D-e, 2D-f. Cases in (a) use the SENKIN method for emulating pressure whereas cases in (b) use the experimental pressure trace. Solid lines indicate cases with both mixture and thermal stratification at $t=0$. Dashed lines indicate cases with only mixture stratification and uniform temperature at $t=0$. The color black represents cases with higher overall equivalence ratio $(\phi=0.69)$, whereas blue represents cases with lower overall equivalence ratio $(\phi=0.52)$. 


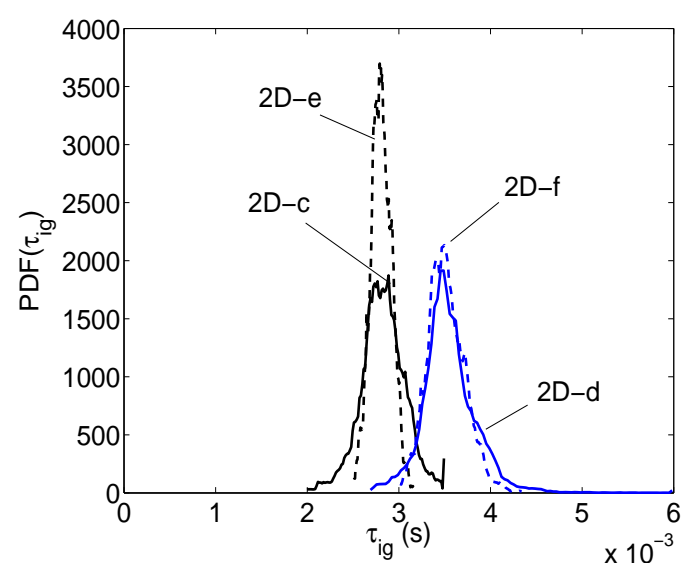

(a)

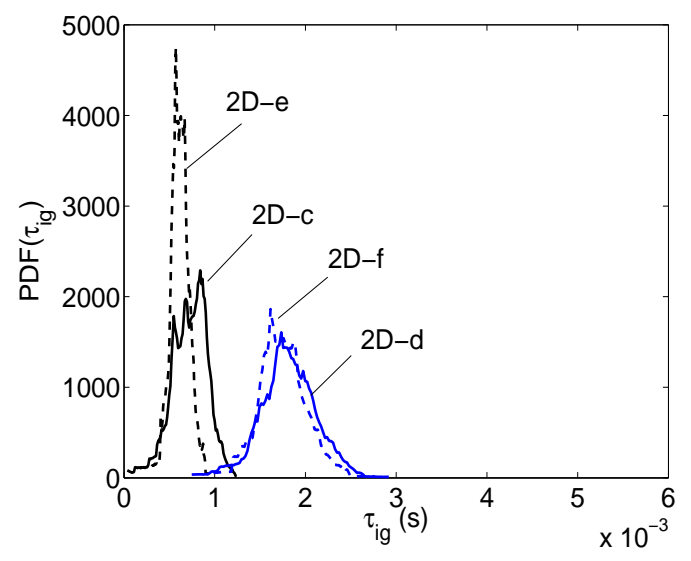

(c)

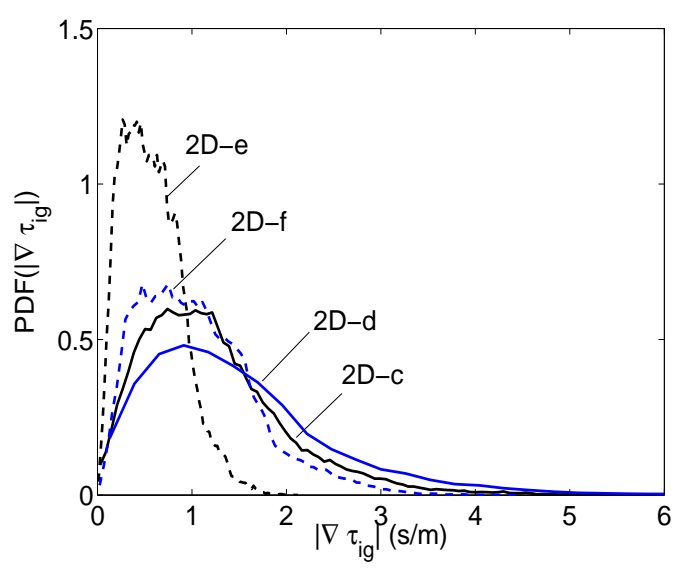

(b)

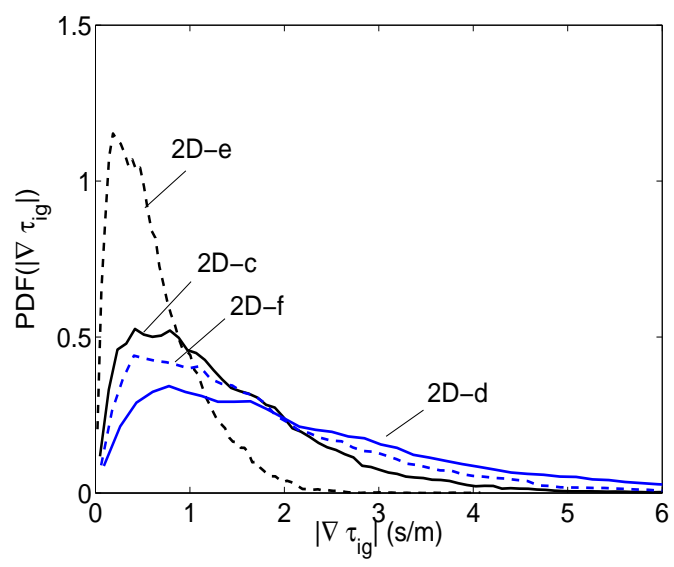

(d)

Figure 19: (a) PDF of the homogeneous ignition delay for cases 2D-c through 2D-f at $t=0 \mathrm{~ms}$ (b) PDF of ignition delay gradient for cases 2D-c through 2D-f at $t=0 \mathrm{~ms}$ (c) PDF of homogeneous ignition delay for cases 2D-c through 2D-f at $t=2.25 \mathrm{~ms}$ (d) PDF of ignition delay gradient for cases 2D-c through 2D-f at $t=2.25 \mathrm{~ms}$. Solid lines indicate cases with both mixture and thermal stratification at $t=0 \mathrm{~ms}$. Dashed lines indicate cases with only mixture stratification and uniform temperature at $t=0 \mathrm{~ms}$. The color black represents cases with higher overall equivalence ratio $(\phi=0.69)$, whereas blue represents cases with lower overall equivalence ratio $(\phi=0.52)$. 
mixture. Large gradients in ignition delay result in flame propagation, whereas small gradients result in spontaneous ignition fronts. Given the considerable extent of flame propagation seen in the cases simulated in the present study, it is of interest to examine the statistics of homogeneous ignition delay. However, in a closed volume system with pressure feedback, the relevant ignition delay times and the gradients are those at the instant a local parcel has reached igniting conditions. These are very different from the initial ignition delays due to both turbulent mixing and compression heating, and hence ignition delays computed from the initial conditions do not characterize the scalar gradient fields when ignition commences. This has been shown in the analytical study by Sankaran and Im [31] and the numerical results of Strozzi et. al. [37], whereby the compression heating from the ignition of hotter parcels causes $\partial \tau_{i g} / \partial T$ in the remaining parcels to be significantly lower compared to constant pressure conditions. The relevant value of $\nabla \tau_{i g}$ depends on the pressure and mixing history and also shows increased sensitivity to composition gradients as,

$$
\nabla \tau_{i g}=\frac{\partial \tau_{i g}}{\partial T} \nabla T+\frac{\partial \tau_{i g}}{\partial Y_{k}} \nabla Y_{k}
$$

where the sensitivity to composition stratification is shown in Figure 4.

0-D ignition delays were computed on a coarser mesh obtained by downsampling the simulation mesh by a factor of four in both directions. The simulation mesh was designed to resolve the smallest radical layers during HTHR, which are typically an order of magnitude thinner than the thermal layers. Gradients in temperature and fuel concentration prior to HTHR were found to be well resolved with this coarser mesh. Given the linear dependence of the ignition delay gradient on gradients in temperature and fuel concentration as seen in Eq. 17, ignition delay gradients are also expected to be well resolved. The homogeneous ignition delay field for this mixture was computed by running a reacting $0-\mathrm{D}$ simulation at 
each point on the coarse mesh. i.e. neglecting any diffusive or convective transport. Each point was however constrained to follow the same pressure history as in the 2-D simulations described in the previous sections. Hence the effect of compression heating was taken into account, whereas mixing effects were neglected.

Figure 19 shows PDFs of ignition delay and magnitude of the gradient of ignition delay at $t=0\left(C A=-20^{\circ} A T D C\right)$ and $t=2.25 m s\left(C A=-4^{\circ} A T D C\right)$, which is subsequent to LTHR for all cases but prior to HTHR. The latter time instant is the latest time at which all cases can reasonably be compared together, since the subsequent HTHR process proceeds at different rates for each of them. Predictably, the two cases with lower n-heptane concentration and overall equivalence ratios have longer ignition delays at both time instances. The range of ignition delay gradients correlates with the range of ignition delays for all cases. In particular, case 2D-e with stratification in n-heptane concentration alone has the narrowest PDF for ignition delay and hence a narrow distribution in ignition delay gradients as well. It is interesting to note that the two cases with higher equivalence ratio (and n-heptane concentration), 2D-c and 2D-e have a narrower range of $\tau_{i g}$ and $\nabla \tau_{i g}$ than the corresponding cases with lower equivalance ratio and n-heptane concentration. This can be inferred from Figure 9, which shows that lower concentrations of n-heptane correspond to a wider range of ignition delays and vice-versa. This also carries over to gradients in ignition delays, since mixtures with a wider range of ignition delays also display a wider range of ignition delay gradients. It can be seen from Figure 19(b) and (d) where cases with lower n-heptane concentration have a wider range of ignition delay gradients (Case 2D-e vs. Case 2D-f and Case 2D-c vs. Case 2D-d). In particular, cases 2D-d and 2D-f with the lower overall equivalence ratios have larger ignition delay gradients than cases 2D-c and 2D-e, respectively. However, those cases exhibit lower fractions of heat release occurring through the flame propagation mode. Hence, based on this limited analysis, the validity of the Zeldovich theory cannot be 
verified for the current set of simulations.

However, there are important caveats to this conclusion. Defining a homogeneous ignition delay field for a general reacting mixture is not a straightforward endeavor. For instance, it could be defined in a constant volume, constant pressure or a multi-zone sense, wherein different parcels do not mix, but do pressure work on each other. Another method is to prescribe a predetermined pressure trace for all points, as was done in the present case. Each of these methods would presumably yield a different result and it is not immediately apparent which method is the most appropriate. Nevertheless, this is a topic of great interest and relevance and will be addressed in more detail in a future study.

\section{Conclusions}

One- and two-dimensional DNS of an RCCI configuration with a gasoline PRF fuel blend have been performed considering parametric variation in n-heptane equivalence ratio, temperature stratification and pressure profile. The one-dimensional simulations show that for uniform fuel concentration, higher initial temperature gradients result in flame propagation whereas lower gradients favor spontaneous ignition fronts. Moreover, even in the absence of initial thermal stratification, i.e. $d T / d x=0$, an initial gradient in n-heptane concentration resulted in premixed flame propagation.

One of the new contributions of this work was the development of a feedback-based algorithm for specifying an arbitrary pressure profile for an igniting mixture. With this new technique for imposing pressure, it was found that zero-dimensional SENKIN based pressure with no feedback from the flow results in pressures much higher than those observed in real engines. Consequently, it was found that for cases with the same initial thermo-chemical conditions but different pressure profiles, a greater fraction of the mixture ignited through 
flame propagation compared to ignition fronts. This was due to the increased ignition delay time associated with the lower pressures.

Similar to the observations from one-dimensional flames noted above, the two-dimensional simulations with uniform temperature also show a substantial fraction of heat release occurring via flame propagation. This fraction was found to be higher for mixtures with a higher concentration of n-heptane. Therefore, it is inferred that high cetane number fuels such as n-heptane with a negative temperature coefficient (NTC) behavior and significant low-temperature chemistry tend to generate heat release mainly through flame propagation (deflagration) compared to low cetane number fuels which favor ignition fronts. Statistics

of ignition delay are computed to assess the Zeldovich theory for mode of combustion propagation based on ignition delay gradients. The data from the current study was found not to support the central thesis of that theory. However, there are several limitations to the analysis presented here and therefore a more detailed assessment of Zeldovich's theory will be presented in a future study. It is hoped that the data generated for this study would be a valuable validation tool for modeling approaches that switch between different models for ignition fronts and deflagration for problems exhibiting multiple modes of combustion.

\section{Acknowledgements}

This research is supported by the Combustion Energy Frontier Research Center (CEFRC), an Energy Frontier Research Center funded by the U.S. Department of Energy (DOE), Office of Science, Office of Basic Energy Sciences (BES) under Award No. DE-SC0001198. Sandia is a multiprogram laboratory operated by Sandia Corporation, a Lockheed Martin Company, for the United States Department of Energy under contract DE-AC04-94AL85000. Computer allocations were awarded by the Department of Energy's Advanced Leadership Computing Challenge (ALCC) at the National Energy Research Scientific Computing Center 
(NERSC) and the INCITE award at the Oak Ridge Leadership Computing Facility (OLCF) at the Oak Ridge National Laboratories (ORNL). This research used resources of the Oak Ridge Leadership Computing Facility at ORNL, which is supported by the Office of Science of the U.S. Department of Energy under Contract No. DE-AC05-00OR22725.

\section{Appendix}

The correlation of Metghalchi et al. [27] is used to calculate the unstrained laminar flame speed, $s_{L}^{0}$,

$$
s_{L}^{0}=s_{L, r e f}^{0}\left(\frac{T_{u}}{T_{u, r e f}}\right)^{\alpha}\left(\frac{P}{P_{r e f}}\right)^{\beta} F_{d i l}
$$

The subscript ref corresponds to reference conditions taken as $298 \mathrm{~K}$ and $1 \mathrm{~atm}$. A scaling factor, $F_{d i l}$ is used to consider the effects of inert species on the laminar flame speed. In this case, since no EGR dilution was used, $F_{d i l}$ was taken to be unity. The exponents, $\alpha$ and $\beta$, used in this correlation are functions of equivalence ratio $\phi$, given by,

$$
\begin{aligned}
& \alpha=2.18-0.8(\phi-1) \\
& \beta=-0.16+0.22(\phi-1)
\end{aligned}
$$

The reference laminar flame speed is calculated using the approach suggested by Yang and Reitz [28] that considers the effect of PRF number (i.e., reactivity) on laminar flame speed.

$$
\begin{gathered}
s_{L, \text { ref }}^{0}=34.1 \phi^{-0.134} \exp \left(-3.86(\phi-1.146)^{2}\right)+ \\
\left(0.13482 \phi-0.09752 \phi^{2}+0.01746 \phi^{3}\right)(100-P R F)
\end{gathered}
$$


The PRF number represents the fraction of iso-octane in the upstream mixture, it is taken to be 0 for $\mathrm{n}$-heptane and 100 for iso-octane. Hence it is computed as $P R F=100 Y_{\text {iso-octane }}$. Since this is a stratified mixture, the equivalence ratio used in the relations above was taken to be the local upstream value of $\phi$ corresponding to the unburnt end along the normal to the flame. 


\section{References}

[1] Manente, V., Tunestal, P., and Johansson, B. "Gasoline Partially Premixed Combustion, the Future of Internal Combustion Engines?" Int. J. Engine Res., 12, 194-208 (2011)

[2] Dec, J. E., Yang ,Y., and Dronniou, N. "Boosted HCCI - Controlling Pressure-Rise Rates for Performance Improvements using Partial Fuel Stratification with Conventional Gasoline." SAE Int. J. Engines, textbf4, 1169-1189 (2011)

[3] Kokjohn, S. L., Hanson, R. M., Splitter, D. A., and Reitz, R. D. "Fuel Reactivity Controlled Compression Ignition (RCCI): A Pathway to Controlled High-Efficiency Clean Combustion." Int. J. Engine. Res., textbf12, 209-226 (2011)

[4] Nieman, D., Dempsey, A., and Reitz, R. D. "Heavy-Duty RCCI Operation using Natural Gas and Diesel." SAE Int. J. Engines, 5, 270-285 (2012)

[5] Splitter, D. A., Hanson, R. M., Kokjohn, S. L., and Reitz, R. D. "Reactivity Controlled Compression Ignition (RCCI) Heavy-Duty Engine Operation at Mid-and High-Loads with Conventional and Alternative Fuels." SAE Tech. Paper, 2011-01-0363 (2011)

[6] Hanson, R. M., Curran, S., Wagner, R., and Reitz, R.D. "Effects of Biofuel Blends on RCCI Combustion in a Light-Duty, Multi-Cylinder Diesel Engine." SAE Int. J. Engines, 6, 488-503 (2013)

[7] Kokjohn, S. L., Musculus, M. P. B., and Reitz, R.D. "Chemiluminescence and Fuel PLIF Imaging of Reactivity Controlled Compression Ignition (RCCI) Combustion." ILASS Americas, 23rd Annual Conference on Liquid Atomization and Spray Systems. (2011) 
[8] Chen, J.H., Choudhary, A., de Supinsky, B., DeVries, M., Hawkes, E.R., Klasky, S., Liao, W.K., Ma, K.L., Mellor-Crummey, J., Podhorszki, N., Sankaran, R., Shende, S., Yoo, C.S. "Terascale direct numerical simulations of turbulent combustion using S3D." Comp. Sci. Disc., 2, 015001 (2009)

[9] Kee, R. J., Rupley, F. M., Miller, J. A., Coltrin, M. E., Grcar, J. F., Meeks, E., Moffat, H. K., Lutz, A. E., Dixon- Lewis, G., Smooke,, M. D., Warnatz, J., Evans, G. H., Larson, R. S., Mitchell, R. E., Petzold, L. R., Reynolds, W. C., Caracotsios, M., Stewart, W. E., Glarborg, P., Wang, C., Adigun, O. CHEMKIN Collection, Release 3.6, Reaction Design, Inc., San Diego, CA (2000)

[10] Kennedy, C.A., Carpenter, M.H. "Several new numerical methods for compressible shear-layer simulations." Appl. Numer. Math., 14, 397-433 (1994)

[11] Kennedy, C.A., Carpenter, M.H., Lewis, R.M. "Low-storage, explicit RungeKutta schemes for the compressible NavierStokes equations." Appl. Numer. Math., 35, 177-219 (2000)

[12] Luong M.B., Luo Z., Lu T.F., Chung S.H., Yoo C.S. "Direct numerical simulations of the ignition of lean primary reference fuel/air mixtures under HCCI condition." Comb. Flame, 16020382047 (2013)

[13] Curran, H. J., Gaffuri, P., Pitz, W.J., Westbrook, C.K. "A Comprehensive Modeling Study of iso-Octane Oxidation." Comb. Flame, 129, 253-280 (2002)

[14] Kokjohn, S., Reitz, R., Splitter, D., and Musculus, M. "Investigation of Fuel Reactivity Stratification for Controlling PCI Heat-Release Rates Using High-Speed Chemiluminescence Imaging and Fuel Tracer Fluorescence" SAE Int. J. Engines, 5, 248-269 (2012)

[15] Peters, N. "Turbulent Combustion" Cambridge University Press (2000) 
[16] Chen, J.H., Hawkes, E.R., Sankaran, R., Mason, S.D., Im, H.G. "Direct numerical simulation of ignition front propagation in a constant volume with temperature inhomogeneities I. Fundamental analysis and diagnostics" Comb. Flame, 145, 128-144 (2006)

[17] Hawkes, E.R., Sankaran, R., Pébay, P.P., Chen, J.H. "Direct numerical simulation of ignition front propagation in a constant volume with temperature inhomogeneities II. Parametric study" Comb. Flame, 145, 145-159 (2006)

[18] Sankaran, R., Im, H.G., Hawkes. E.R., Chen, J.H. "The effects of non-uniform temperature distribution on the ignition of a lean homogeneous hydrogen-air mixture." Proc. Comb. Inst., 30, 875-882 (2005)

[19] Sankaran, R., Hawkes, E.R., Yoo, C.S., Chen, J.H., Lu, T., Law, C.K. "Direct numerical simulation of stationary lean premixed methane-air flames under intense turbulence." Proc. 5th U.S. Comb. Meet., Paper B09, San Diego (2007)

[20] Lu, T., Yoo, C.S., Chen, J.H., Law, C.K. "Three-dimensional direct numerical simulation of a turbulent lifted hydrogen flame in heated coflow: a chemical explosive mode analysis." J. Fluid Mech., 652, 45-64 (2010)

[21] Shan, R., Yoo, C.S., Chen, J.H., Lu, T. "Computational diagnostics for n-heptane flames with chemical explosive mode analysis." Comb. Flame, 159, 3119-3127 (2012)

[22] Bhagatwala, A., Chen, J.H., Lu, T. "Direct numerical simulations of SACI/HCCI with ethanol." Comb. Flame, 161, 1826-1841 (2014)

[23] Bansal, G., Im, H.G "Autoignition and front propagation in low temperature combustion engine environments." Comb. Flame, 158, 2105-2112 (2011) 
[24] Yoo, C.S., Lu, T., Chen, J.H., Law, C.K. "Direct numerical simulations of ignition of a lean n-heptane/air mixture with temperature inhomogeneities at constant volume: Parametric study." Comb. Flame, 158, 1727-1741 (2011)

[25] Dai, P., Chen, Z., Chen, S., Ju, Y. "Numerical experiments on reaction front propagation in n-heptane/air mixture with temperature gradient." Proc. Comb. Inst., DOI: 10.1016/j.proci.2014.06.102 (2014)

[26] Law, C.K. "Combustion Physics" Cambridge Press (2006)

[27] Metghalchi, M., Keck, J. C "Burning Velocities of Mixtures of Air with Methanol, Isooctane, and Indolene at High Pressures and Temperatures." Comb. Flame, 48, 191$210(1982)$

[28] Yang, S., Reitz ,R. D. "Integration of a Continuous Multi-Component Fuel Evaporation Model with an Improved G-Equation Combustion and Detailed Chemical Kinetics Model with Application to GDI Engines." SAE Technical Paper, 2009-01-0722 (2009)

[29] Domingo, P., Vervisch, L. "Triple flames and partially premixed combustion in autoignition of non-premixed turbulent mixtures." International Symposium on Combustion, $\mathbf{2 6}$, 233-240 (1996)

[30] Heywood, J.B. "Internal combustion engine fundamentals" Mcgraw-Hill New York (1988)

[31] Sankaran, R., Im, H.G., Hewson, J.C. "Analytical model for autoignition in a thermally stratified HCCI engine." Comb. Sci. Tech., 179, 1963-1989 (2007)

[32] Lorensen, W. E., Harvey, E. C. "Marching cubes: A high resolution 3D surface construction algorithm." ACM Siggraph Computer Graphics, 21 (1987) 
[33] Tamagna, D., Gentili, R., Ra, Y., Reitz, R. "Multidimensional Simulation of the Influence of Fuel Mixture Composition and Injection Timing in Gasoline-Diesel Dual-Fuel Applications." SAE Technical Paper, 2008-01-0031 (2008)

[34] Zel'dovich, Y.B. "Regime classification of an exothermic reaction with non-uniform initial conditions." Comb. Flame, 39, 211 (1980)

[35] Linan, A., Williams, F.A. "Autoignition of non-uniform mixtures in chambers of variable volume." Comb. Sci. Tech., 105, 245-263 (1995)

[36] Seshadri, K., Bollig, M., Peters, N. "Numerical and asymptotic studies of the structures of stoichiometric and lean premixed heptane flames." Comb. Flame, 108, 518-536 (1997)

[37] Strozzi, C., Mura, A., Sotton, J. and Bellenoue, M. "Experimental analysis of propagation regimes during the autoignition of a fully premixed methane-air mixture in the presence of temperature inhomogeneities." Comb. Flame, 159 3323-3341 (2012)

[38] Chen, J. H., Mason, S. D., Hewson, J. C. "The effect of temperature inhomogeneity on low-temperature autoignition of fuel-lean premixed hydrogen/air mixtures." Proc. of the 3rd Joint Meeting of the U.S. sections of the Comb. Inst., B30 (2003). 